\title{
Path-integral formulation of closed strings
}

\author{
Shyamoli Chaudhuri, Hikaru Kawai, and S.-H. Henry Tye \\ Newman Laboratory of Nuclear Studies, Cornell University, Ithaca, New York 14853
}

(Received 21 April 1986)

\begin{abstract}
We construct the covariant path integral for the Neveu-Schwarz-Ramond superstring in superspace, with manifest invariance under diffeomorphisms and local supersymmetry transformations. Spin structure is introduced, and the constraints imposed by modular invariance on fermionic string models examined. The critical exponents of the bosonic and fermionic string models in space-time dimensions less than the critical dimension are obtained for world surfaces of arbitrary topology.
\end{abstract}

\section{INTRODUCTION}

Closed-string models are described by a twodimensional field theory on the world sheet, a multiply connected two-dimensional manifold embedded in spacetime. A closed Riemann surface with $h$ handles, or Euler characteristic $\chi=2-2 h$, corresponds to an $h$ th-order loop diagram. The path-integral formulation provides a natural framework for the study of multiloop amplitudes. Such multiloop diagrams involve a complicated dependence of the functional integrals on the moduli, and for superstring models the supermoduli, of the Riemann surfaces.

Following Polyakov's path-integral formulation ${ }^{1}$ for strings, the importance of the moduli was first pointed out by Friedan ${ }^{2}$ and subsequently analyzed by Alvarez. ${ }^{3}$ More recently the structure of moduli space, or its covering space Teichmueller space, has been further clarified by Polchinski, ${ }^{4}$ Moore and Nelson, ${ }^{5}$ and D'Hoker and Phong. ${ }^{6}$ For the fermionic string, ${ }^{7}$ some progress has been made by Martinec, ${ }^{8}$ Moore, Nelson, and Polchinski, ${ }^{9}$ and others. $^{10}$

In this work, we construct a covariant path-integral formulation of the fermionic string ${ }^{11}$ in superspace and extend it to include spin structure. Intuitively, the functional integration of fermions in the path integral must include a weighted sum over the possible boundary conditions for the spinor fields, periodic and antiperiodic, allowed by the symmetries of the fermionic string model. However, the allowed sums are severely restricted by modular invariance. For example, at the one-loop level we obtain the space-time supersymmetry projection ${ }^{12}$ of the Neveu-Schwarz-Ramond (NSR) model. ${ }^{11}$ For the heterotic string ${ }^{13}$ the gauge groups $\mathrm{SO}(32)$ and $\mathrm{E}_{8} \otimes \mathrm{E}_{8}$ also arise from the requirement of modular invariance. Generalizations of these arguments put constraints on new string models.

Both the NSR and the heterotic string actions can be expressed either in terms of component fields, ${ }^{14}$ or superfields. ${ }^{15}$ The superspace treatment of fermionic strings is an elegant and succinct parallel to the bosonic construction. An advantage of the covariant formulation in superspace is that invariance under diffeomorphisms and local supersymmetry transformations is manifest. For the heterotic and other string models having world-sheet chiral fields, the measure in the path integral cannot be defined in a Lorentzinvariant way. This can give rise to Lorentz, or local gravitational, anomalies. The absence of such an anomaly forces these string models to the critical dimension. Consequently, our discussion of these models is restricted to ten dimensions.

To clarify the meaning of this admittedly abstract formalism we obtain explicit expressions for the partition functions at the one-loop level. The meaning of the conformal Killing vectors (spinors), the moduli (supermoduli), and the spin structure, as well as the powerful constraints coming from modular invariance become apparent.

The classical string actions all have local conformal invariance. However, when the dimensionality of spacetime is less than $D_{\text {critical }}$ a conformal anomaly is introduced at the quantum level and we must integrate over the Liouville degree of freedom. ${ }^{1,7}$ In such cases, we can rewrite the partition functions in the form ${ }^{16}$

$$
\begin{aligned}
& Z=\sum_{\chi} Z_{\chi}, \\
& Z_{\chi}=\int_{0}^{\infty} d A F_{\chi}(A),
\end{aligned}
$$

and consider its behavior at fixed area $A$. Asymptotical$1 y$,

$$
F_{\chi}(A) \underset{A \rightarrow \infty}{\sim} C_{\chi} \quad A^{b \chi} e^{K A},
$$

where $b_{\chi}$ is a critical exponent and $K$ is a regularizationdependent quantity. For the bosonic string, the critical exponent for surfaces of spherical topology $b_{2}$ has been calculated by Zamolodchikov. ${ }^{17} \mathrm{We}$ generalize this calculation for arbitrary topology with the result

$$
b_{\chi}=\frac{\chi}{12}(D-19)-1,
$$

where we make use of the theorem on the existence of a constant-curvature metric for any topology. An analogous calculation $^{17}$ yields the Hausdorff dimension of the surfaces. All the other critical exponents can be obtained in terms of these two. The critical exponent $b_{\chi}$ can be calculated for a variety of string models with an arbitrary conformal field theory defined on the world sheet, an outstanding exception being the heterotic strings. The re- 
quired cancellation of local gravitational anomalies forces them to the critical dimension with conformal invariance intact.

For string models having world-sheet supersymmetry, in space-time dimensions below their critical dimension, we can introduce the following substitution:

$$
Z_{\chi}=\int_{0}^{\infty} d L F_{\chi}(L)
$$

where $L$ is a characteristic length scale of the Riemann surface. Asymptotically,

$$
F_{\chi}(L) \underset{L \rightarrow \infty}{\sim} C_{\chi} L^{b} \chi e^{K L},
$$

and the critical exponent is calculated to be

$$
b_{\chi}=\frac{\chi}{8}(D-9)-1 \text {. }
$$

The evaluation of $b_{\chi}$ naturally suggests the (world-sheet) superspace generalization of the theorem on the existence of a unique constant curvature metric on a Riemann surface of arbitrary topology.

In Sec. II we begin by reviewing the path-integral formulation of the bosonic string ${ }^{3-6}$ to establish our notation. Choosing the space of constant curvature metrics as a fiducial gauge slice we calculate the critical exponent $b_{\chi}$ for an arbitrary value of the Euler characteristic $\chi$. In Sec. III we consider the consequences of placing fermions on the world sheet, and introduce the summation over spin structures in the partition function. The construction of the path integral for the NSR superstring in superspace is carried out in Sec. IV. In Sec. V we approach the problem of constructing a path-integral formulation for the heterotic string in a chiral superspace.

The torus is analyzed in depth in Sec. VI, clarifying the meaning of spin structure and modular invariance. The bosonic string torus is evaluated to illustrate the calculation of the critical exponent and the tori for the superstring and the heterotic string are calculated to illustrate spin structure and the constraints coming from modular invariance.

Our calculations are entirely done in two-dimensional Euclidean space. Appendix A contains our definition of Majorana-Weyl fermions in two-dimensional Euclidean space. Appendix B reviews some of the relevant facts ${ }^{15}$ about two-dimensional superspace used in Secs. IV and V.

\section{THE BOSONIC STRING AND ITS CRITICAL EXPONENTS}

We begin this section with a review of the path-integral formulation for closed Riemann surfaces, ${ }^{3,5,6}$ and then evaluate the critical exponents of the bosonic string. The partition function is formally given by

$$
Z=\sum_{\text {topologies }} \int_{\mathrm{m} \otimes R^{D}} \mathrm{D} g_{m n} \mathrm{D} X^{I} \exp (-S[X, g]),
$$

where $\mathbf{m}$ is the space of metrics, and $R^{D}$ the space of all embeddings of the world sheet in a $D$-dimensional Euclidean space-time. $X^{I}(\xi), I=1, \ldots, D$, and $g_{m n}(\xi)$, $m, n=1,2$, are two-dimensional scalar and tensor fields defined on a differentiable manifold $M$. The action is

$$
S\left[X, g_{m n}\right]=\int_{M} d^{2} \xi \sqrt{g} g^{m n} \partial_{m} X^{I} \partial_{n} X^{I},
$$

and the volume elements in function space, $\mathbf{D} X$ and $\mathbf{D} g_{m n}$, are defined by the metric

$$
\left\|\delta X^{I}\right\|^{2}=\int d^{2} \xi \sqrt{g}\left(\delta X^{I}\right)^{2}
$$

and

$\left\|\delta g_{m n}\right\|^{2}=\int d^{2} \xi \sqrt{g}\left(g^{m n} g^{p q}+C g^{m p} g^{n q}\right) \delta g_{m p} \delta g_{n q}$.

$C$ is an arbitrarily chosen constant which, for convenience, we set equal to zero.

Both the action and the measure of the path integral are invariant under the group of diffeomorphisms $\{D\} . A$ diffeomorphism is a differentiable one-to-one mapping of $M$ onto itself,

$$
\xi^{m} \in M \rightarrow \xi^{m^{\prime}} \in M
$$

under which $X(\xi)$ and $g_{m n}(\xi)$ transform as follows:

$$
\begin{aligned}
& X(\xi) \rightarrow X^{\prime}(\xi), \quad X^{\prime}\left(\xi^{\prime}\right)=X(\xi) \\
& g_{m n}(\xi) \rightarrow g_{m n}^{\prime}(\xi), \\
& g_{m n}^{\prime}\left(\xi^{\prime}\right) d \xi^{m^{\prime}} d \xi^{n^{\prime}}=g_{m n}(\xi) d \xi^{m} d \xi^{n} .
\end{aligned}
$$

Under a conformal transformation, the fields transform as

$$
\begin{aligned}
& X(\xi) \rightarrow X(\xi) \quad \text { (no change) } \\
& g_{m n}(\xi) \rightarrow g_{m n}^{\prime}(\xi)=\Lambda(\xi) g_{m n}(\xi) .
\end{aligned}
$$

The definitions of the metrics in (2.3) and (2.4) are not invariant under the action of the conformal group $\{C\}$. This is the origin of the conformal anomaly. Taking into account the invariance of the partition function under $\{\mathrm{D}\},(2.1)$ can be rewritten as

$$
Z=\int \mathbf{D} X^{I} \frac{\mathbf{D} g_{m n}}{\mathbf{\Omega}_{g}(\{\mathrm{D}\})} \exp (-S[X, g]),
$$

where $\boldsymbol{\Omega}_{g}(\{\mathbf{D}\})$ is a formal expression for the volume of the diffeomorphism group evaluated at $g_{m n}$. It is convenient for our purposes to factor out the volume of the subgroup $\left\{\mathbf{D}_{0}\right\}$ of diffeomorphisms that are continuously connected to the identity. In general, if we consider leftright-asymmetric theories, anomalies can appear associated with the group $\{D\}$. The anomaly associated with $\left\{D_{0}\right\}$ is the local gravitational anomaly, while that associated with the mapping class group, $G \equiv\{D\} /\left\{D_{0}\right\}$, is the modular anomaly. Formally, the volume of the invariance group can be written as

$$
\boldsymbol{\Omega}_{g}(\{\mathrm{D}\})=\boldsymbol{\Omega}_{g}\left(\left\{\mathrm{D}_{0}\right\}\right)|\boldsymbol{G}|,
$$

where $|G|$ is the order of the discrete group $G$. In principle, $\boldsymbol{\Omega}_{g}(\{\mathrm{D}\})$ is fixed once the metric for infinitesimal diffeomorphisms is defined:

$$
\left\|\delta \eta^{m}\right\|^{2}=\int d^{2} \xi \sqrt{g} g_{m n} \delta \eta^{m} \delta \eta^{n}
$$


where $\delta \eta^{m}$ generates infinitesimal diffeomorphisms:

$$
\xi^{m} \rightarrow \xi^{m}+\delta \eta^{m}
$$

In order to make the meaning of $\mathbf{D} g / \boldsymbol{\Omega}_{g}(\{\mathrm{D}\})$ more transparent, we introduce a gauge slice $S^{\prime}$ which represents $\mathbf{m}^{\prime} \equiv \mathbf{m} /\left\{\mathbf{D}_{0}\right\}$, a slice in the functional space of all metrics $\mathbf{m}$ that is transverse to the action of the group $\left\{D_{0}\right\}$. The space $\mathbf{m} /\{\mathbf{C}\} \otimes\left\{D_{0}\right\}$ is called Teichmueller space and is known to be finite dimensional. If we fix a representation of Teichmueller space $S \equiv\left\{\hat{g}_{m n}(\xi, \tau)\right\}$, any element on the gauge slice $S^{\prime}$ can be expressed as

$$
g_{m n}(\xi)=e^{\phi(\xi)} \widehat{g}_{m n}\left(\xi, \tau_{i}\right),
$$

where the $\tau_{i}$ are $c$ numbers that parametrize the space $S$. Let $\delta g$ be an element of $T_{g} \mathrm{~m}$, the tangent space of the manifold $m$ at any $g \in S^{\prime}$. An arbitrary infinitesimal variation $\delta g \in T_{g} \mathbf{m}$ (see Fig. 1) can be expressed as

$$
\begin{aligned}
\delta g_{m n}(\xi)= & \delta \phi(\xi) e^{\phi(\xi)} \hat{g}_{m n}(\xi, \tau)+e^{\phi(\xi)} \frac{\partial \widehat{g}_{m n}}{\partial \tau_{i}}(\xi, \tau) \delta \tau_{i} \\
& +\left(\nabla_{m} \delta \eta_{n}+\nabla_{n} \delta \eta_{m}\right),
\end{aligned}
$$

where $\nabla_{m}$ is the covariant derivative on the world sheet. The first two terms are variations within the gauge slice $S^{\prime}$, while the last term represents the effect of an infinitesimal diffeomorphism operating on $g_{m n}(\xi)$. In order to avoid redundant parameters, conformal Killing vectors should be excluded from the $\delta \eta^{m}$, as is done explicitly later in this section.

We now introduce an orthogonal decomposition of $T_{g}$ m as follows:

$$
T_{g} \mathbf{m}=G \oplus \operatorname{Im} P_{1} \oplus \operatorname{ker} P_{1}^{\dagger} .
$$

$G$ is the set of all infinitesimal, symmetric, rank-2 tensor fields that can be expressed in the form $\delta \lambda(\xi) g_{m n}(\xi)$, where $\delta \lambda(\xi)$ is an infinitesimal scalar field. The operator $P_{1}$ maps infinitesimal vector fields into symmetric, traceless, rank-2 tensors: ${ }^{3}$

$$
P_{1}: \quad V_{1} \rightarrow V_{2}
$$

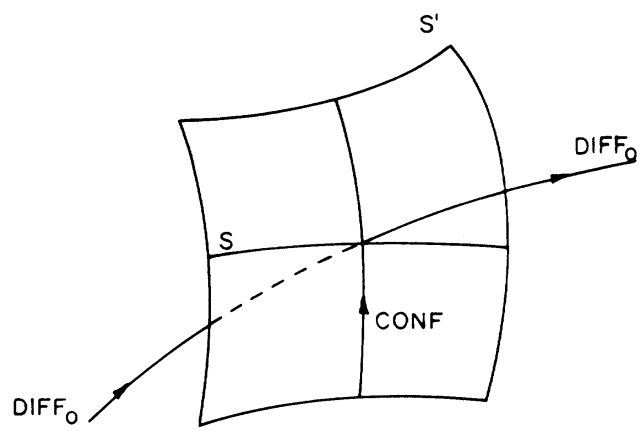

FIG. 1. $S^{\prime}$ is a gauge slice which represents $\mathbf{m}^{\prime}=\mathbf{m} /\left\{\mathbf{D}_{0}\right\}$ where $\mathbf{m}$ is the space of metrics. In the space of $\mathbf{m}^{\prime}$, the gauge slice $S$ represents Teichmueller space, $\mathbf{m} /\left\{\mathrm{D}_{0}\right\} \otimes\{\mathrm{C}\}$. and

$$
V_{1}=\left\{\delta \eta^{m}\right\}, \quad V_{2}=\left\{\delta h_{m n} ; g^{m n} \delta h_{m n}=0\right\} .
$$

Explicitly,

$\left(P_{1} \delta \eta\right)_{m n}=\nabla_{m} \delta \eta_{n}+\nabla_{n} \delta \eta_{m}-g_{m n} \nabla^{p} \delta \eta_{p}$

Since both $\delta \eta_{m}$ and $\delta h_{m n}$ have two components each, and $P_{1}$ is a nondegenerate elliptic operator, it follows that the kernels of the operators $P_{1}$, and its adjoint $P_{1}^{\dagger}$, are finite dimensional. It is easy to check that $G, \operatorname{Im} P_{1}$, and $\operatorname{ker} P_{1}^{\dagger}$ are orthogonal to each other. This is schematically illustrated in Fig. 2, where $\delta \phi$ lies along the axis of $G ; \delta \eta^{m}$ is in the plane of $\operatorname{Im} P_{1}$ and $G$; and $\delta \tau_{i}$ lies in the "parallelopiped" $\left(G \oplus \operatorname{Im} P_{1} \oplus \operatorname{ker} P_{1}^{+}\right)$. Since $G \oplus \operatorname{Im} P_{1}$ covers all directions swept out by $\{C\} \otimes\left\{D_{0}\right\}$, the dimension of the remaining space, $\operatorname{ker} P_{1}^{\dagger}$, must be the same as the dimension of Teichmueller space: $\operatorname{dim} \operatorname{ker} P_{1}^{+}=\operatorname{dim} S$. Then $(2.15)$ can be rewritten as

$$
\delta g_{m n}(\xi)=\delta \lambda(\xi) g_{m n}(\xi)+\delta h_{m n}(\xi)+\sum_{i} \delta c_{i} \psi_{m n}^{(i)}
$$

where $\left\{\psi_{m n}^{(i)}\right\}$ is a basis for $\operatorname{ker} P_{1}^{\dagger}$, and the $\delta c_{i}$ 's are infinitesimal parameters. Comparing this expression with (2.15) gives

$$
\left(\begin{array}{c}
\delta \lambda(\xi) \\
\delta h_{m n}(\xi) \\
\delta c_{i}
\end{array}\right)=\left(\begin{array}{ccc}
1 & * & * \\
0 & P_{1} & * \\
0 & 0 & T^{i j}
\end{array}\right)\left(\begin{array}{c}
\delta \phi(\xi) \\
\delta \eta_{m}(\xi) \\
\delta \tau_{j}
\end{array}\right),
$$

where

$$
\left\langle\psi^{(i)} \mid \psi^{(l)}\right\rangle T_{l}^{j}=\left\langle\psi^{(i)} \mid e^{\phi(\xi)} \frac{\partial \widehat{g}_{m n}}{\partial \tau_{j}}\right\rangle .
$$

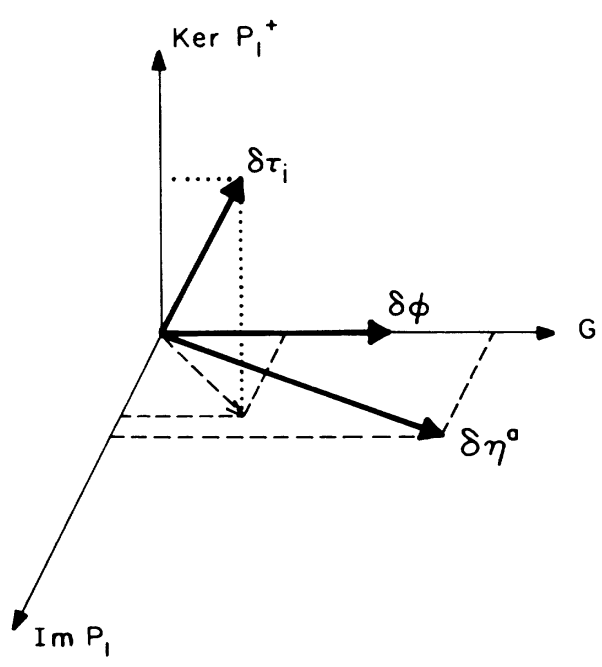

FIG. 2. A schematic picture illustrating the variations $\delta g$ in the tangent space $T_{g} \mathbf{m}$ of metrics $g \in S^{\prime}$ where $T_{g} \mathbf{m}=G \oplus \operatorname{Im} P_{1} \oplus \operatorname{Ker} P_{1}^{\dagger}$. 
The volume element $\mathbf{D} g$ is easily expressed in terms of $\delta \lambda(\xi), \delta h_{m n}(\xi)$, and $\delta c_{i}$ since the spaces $G, \operatorname{Im} P_{1}$, and $\operatorname{ker} P_{1}^{\dagger}$ are mutually orthogonal. Explicitly,

$$
\mathbf{D} g=\mathbf{D} \lambda \mathbf{D} h \prod_{i} \delta c_{i}\left(\operatorname{det}\left\langle\psi^{(i)} \mid \psi^{(j)}\right\rangle\right)^{1 / 2},
$$

where $\mathbf{D} \lambda$ and $\mathbf{D} h$ must be defined by metrics obtained by restricting the metric in (2.4) to the spaces $G$ and $\operatorname{Im} P_{1}$, respectively. Since the transformation matrix $(2.21)$ is triangular, the Jacobian of the transformation from $\left(\mathbf{D} \lambda \mathbf{D} h \prod_{i} \delta c_{i}\right)$ to $\left(\mathbf{D} \phi \mathbf{D} \eta \prod_{i} d \tau_{i}\right)$ is easily evaluated:

$$
\mathbf{D} g=\frac{\mathbf{D} \phi\left(\operatorname{det}^{\prime} P_{1}^{\dagger} P_{1}\right)^{1 / 2} \mathbf{D}^{\prime} \eta \operatorname{det}\left\langle\psi^{(i)} \mid e^{\phi(\xi)} \frac{\partial \widehat{g}_{m n}}{\partial \tau_{j}}\right\rangle \prod_{i} d \tau_{i}}{\left(\operatorname{det}\left\langle\psi^{(i)} \mid \psi^{(j)}\right\rangle\right)^{1 / 2}} .
$$

The primes in $\left(\operatorname{det}^{\prime} P_{1}^{\dagger} P_{1}\right)^{1 / 2}$ and $\mathbf{D}^{\prime} \eta$ mean that we should exclude the conformal Killing vectors. In other words, $\mathbf{D}^{\prime} \eta$ denotes the measure for $\left(\operatorname{ker} P_{1}\right)^{\perp}$. Finally, consider the volume of the gauge group $\boldsymbol{\Omega}_{g}(\{\mathrm{D}\})=|G| \boldsymbol{\Omega}_{g}\left(\left\{\mathbf{D}_{0}\right\}\right)$. The volume element $\mathbf{D} \eta$ is defined by the metric in $(2.12)$ and can be decomposed into $\operatorname{ker} P_{1}$ and $\left(\operatorname{ker} P_{1}\right)^{\perp}$ :

$$
\mathbf{D} \eta=\mathbf{D}^{0} \eta \mathbf{D}^{\prime} \eta
$$

Thus,

$$
\frac{\mathbf{D} g}{\boldsymbol{\Omega}_{g}\left(\left\{\mathbf{D}_{0}\right\}\right)}=\frac{\mathbf{D} \phi\left(\operatorname{det}^{\prime} P_{1}^{\dagger} P_{1}\right)^{1 / 2} \operatorname{det}\left\langle\psi^{(i)} \mid e^{\phi} \frac{\partial \widehat{g}_{m n}}{\partial \tau_{j}}\right\rangle}{\boldsymbol{\Omega}_{g}\left(\left\{\mathbf{C K}_{g}\right\}\right)\left(\operatorname{det}\left\langle\psi^{(i)} \mid \psi^{(j)}\right\rangle\right)^{1 / 2}} \boldsymbol{\Pi}_{i} d \tau_{i} .
$$

$\mathbf{\Omega}_{g}\left(\left\{\mathbf{C K}_{g}\right\}\right)$ is the volume of the subgroup of $\left\{\mathrm{D}_{0}\right\}$ generated by the conformal Killing vectors. If $\boldsymbol{M}$ is topologically equivalent to a sphere, there are six conformal Killing vectors, and $\left\{\mathrm{CK}_{g}\right\}=\mathrm{SL}(2, C)$. The volume of conformal Killing vector space is then infinite. If $M$ is a torus, there are two conformal Killing vectors, $\left\{C K_{g}\right\}=\mathbf{U}(1) \otimes \mathbf{U}(1)$, and $\boldsymbol{\Omega}_{g}\left(\left\{\mathrm{CK}_{g}\right\}\right)$ is finite. For any higher genus $(\chi=-2,-4,-6, \ldots)$ there are no conformal Killing vectors. We can now evaluate the partition function in (2.10):

$$
Z=\int \frac{\mathbf{D} \phi\left(\operatorname{det}^{\prime} P_{1}^{\dagger} P_{1}\right)^{1 / 2} \operatorname{det}\left\langle\psi^{(i)} \mid e^{\phi} \frac{\partial \widehat{\mathrm{g}}_{m n}}{\partial \tau_{j}}\right\rangle \prod_{i} d \tau_{i}}{\mathbf{\Omega}_{g}\left(\left\{\mathbf{C K}_{g}\right\}\right)\left(\operatorname{det}\left\langle\psi^{(i)} \mid \psi^{(j)}\right\rangle\right)^{1 / 2}} \mathbf{D} X^{I} \exp (-S[X, g]) .
$$

The functional integral over $X^{I}$ gives the factor

$$
\left(\operatorname{det}^{\prime} \Delta_{g}\right)^{-D / 2} V\left(\int \sqrt{g} d^{2} \xi\right]^{D / 2}
$$

where the system is put in a periodic box of volume $V$, and the prime implies that the zero modes of the Laplacian $\Delta_{g}$ are excluded. Using the heat-kernel method, ${ }^{3}$ we can calculate the variation of det $^{\prime} \Delta_{g}$ under a conformal transformation. Let us introduce the vacuum functional

$$
W\left[g_{m n}\right]=\ln \left[\left(\operatorname{det}^{\prime} \Delta_{g}\right)^{-1 / 2}\left[\int \sqrt{g} d^{2} \xi\right]^{1 / 2}\right]
$$

The variation under infinitesimal conformal transformations is given by

$$
\begin{aligned}
\delta W & =W\left[e^{-\delta \phi} g_{m n}\right]-W\left[g_{m n}\right] \\
& =\frac{c}{48 \pi} \int \sqrt{g} R(g) \delta \phi d^{2} \xi .
\end{aligned}
$$

$R(g)$ is the scalar curvature calculated from $g_{m n}$, and $c$ is the central charge of the Virasoro algebra for a free scalar field. Using the equation

$$
R\left(e^{-\phi} g\right)=e^{\phi} R(g)-e^{\phi} \Delta_{g} \phi,
$$

(2.29) can be integrated to give

$$
W\left[e^{-\phi} g_{m n}\right]=W\left[g_{m n}\right]+\frac{c}{48 \pi} \int d^{2} \xi\left(\frac{1}{2} \sqrt{g} g^{m n} \partial_{m} \phi \partial_{n} \phi+\sqrt{g} R \phi\right)
$$

Thus, on the gauge slice $S^{\prime}=\left\{g ; g_{m n}=\mathrm{e}^{-\phi} \widehat{g}_{m n}\right\}$, 


$$
\begin{aligned}
V\left(\operatorname{det}^{\prime} \Delta_{g}\right)^{-D / 2}\left[\int \sqrt{g} d^{2} \xi\right]^{D / 2}= & V \exp \left[\frac{D}{48 \pi} \int d^{2} \xi\left(\frac{1}{2} \sqrt{\hat{g}} \hat{g}^{m n} \partial_{m} \phi \partial_{n} \phi+\sqrt{\hat{g}} \hat{R} \phi\right)\right) \\
& \times\left(\operatorname{det}^{\prime} \Delta_{\hat{g}}\right)^{-D / 2}\left[\int \sqrt{\hat{g}} d^{2} \xi\right]^{D / 2}
\end{aligned}
$$

Again, we can use the heat-kernel method to evaluate the Faddeev-Popov determinants:

$$
\begin{aligned}
\prod_{i} d \tau_{i} \frac{\left(\operatorname{det}^{\prime} P_{1}^{\dagger} P_{1}\right)_{g}^{1 / 2} \operatorname{det}\left\langle\psi^{(i)} \mid e^{-\phi} \frac{\partial \hat{g}_{m n}}{\partial \tau_{j}}\right\rangle_{g}}{\mathbf{\Omega}_{g}\left(\left\{\mathbf{C K}_{g}\right\}\right)\left(\operatorname{det}\left\langle\psi^{(i)} \mid \psi^{(j)}\right\rangle_{g}\right)^{1 / 2}}= & {\left[\frac{\prod_{i} d \tau_{i} \operatorname{det}\left\langle\psi^{(i)} \mid \frac{\partial \hat{g}}{\partial \tau_{j}}\right\rangle_{\hat{g}}}{\left(\operatorname{det}\left\langle\psi^{(i)} \mid \psi^{(j)}\right\rangle_{\hat{g}}\right)^{1 / 2}} \frac{\left(\operatorname{det}^{\prime} \hat{P}^{\dagger} \hat{P}\right)^{1 / 2}}{\Omega_{\hat{g}}\left\{\mathbf{C K}_{\hat{g}}\right\}}\right] } \\
& \times \exp \left[\frac{-26}{48 \pi} \int d^{2} \xi\left(\frac{1}{2} \sqrt{\hat{g}} \hat{g}^{m n} \partial_{m} \phi \partial_{n} \phi+\sqrt{\hat{g}} \hat{R} \phi\right)\right] .
\end{aligned}
$$

Notice that the $\phi$ dependence in $(2.33)$ is confined to the exponential.

The factor in the first set of large parentheses in (2.33), namely,

$$
\left(\frac{\prod_{i} d \tau_{i}\left\langle\operatorname{det}\left\langle\psi^{(i)} \mid \frac{\partial \hat{g}}{\partial \tau_{j}}\right\rangle_{\hat{g}}\right)}{\left(\operatorname{det}\left\langle\psi^{(i)} \mid \psi^{(j)}\right\rangle_{\hat{g}}\right)^{1 / 2}} \frac{\left(\operatorname{det}^{\prime} \hat{P}^{\dagger} \hat{P}\right)^{1 / 2}}{\boldsymbol{\Omega}_{\hat{g}}\left(\left\{\mathbf{C K}_{\hat{g}}\right\}\right)}\right),
$$

and the combination $\left(\operatorname{det}^{\prime} \Delta_{\hat{g}} / \int \sqrt{\hat{g}} d^{2} \xi\right)^{-D / 2}$ are invariant under modular transformations, $G=\{\mathrm{D}\} /\left\{\mathrm{D}_{0}\right\}$. Thus we can absorb $1 /|G|$ in the measure to obtain an integral defined in moduli space: $\mathbf{m} /\{\mathrm{C}\} \otimes\{\mathrm{D}\}$. Combining the results (2.32) and (2.33), the partition function becomes

$$
\begin{aligned}
Z=V \int[d \tau] & {\left[\left(\operatorname{det}^{\prime} \hat{\boldsymbol{P}}^{\dagger} \hat{\boldsymbol{P}}\right)^{1 / 2}\left[\frac{\operatorname{det}^{\prime} \Delta_{\hat{g}}}{\int \sqrt{\hat{\mathrm{g}}} d^{2} \xi}\right]^{-D / 2}\right.} \\
& \left.\times \frac{1}{\mathbf{\Omega}_{\hat{g}}\left\{\mathbf{C K}_{\hat{g}}\right\}}\right] \int \mathbf{D} \phi e^{-[(26-D) / 48 \pi] S_{L}[\phi]},
\end{aligned}
$$

where $S_{L}$ is the Liouville action

$$
S_{L}=\int\left(\frac{1}{2} \sqrt{\hat{g}} \hat{g}^{m n} \partial_{m} \phi \partial_{n} \phi+\sqrt{\hat{g}} \hat{R} \phi\right) d^{2} \xi \text {. }
$$

Here $[d \tau]$ is defined as

$$
[d \tau] \equiv\left(\frac{\prod_{i} d \tau_{i} \operatorname{det}\left\langle\psi^{(i)} \mid \frac{\partial \hat{g}}{\partial \tau_{j}}\right\rangle_{\hat{g}}}{\left(\operatorname{det}\left\langle\psi^{(i)} \mid \psi^{(j)}\right\rangle_{\hat{g}}\right)^{1 / 2}}\right) .
$$

Henceforth, we denote the factor in the large parentheses in (2.34) by $\Delta(\tau)$.

Let us now discuss the asymptotic behavior of the number of random surfaces of a given topology ${ }^{16,17}$ and fixed area $A$. This is defined by

$$
\begin{aligned}
F_{\chi}(A)= & \int \frac{\mathbf{D} X \mathbf{D} g}{\Omega_{g}(\{\mathbf{D}\})} \\
& \times \exp (-S[X, g]) \delta\left[\int \sqrt{g} d^{2} \xi-A\right] \\
= & V \int[d \tau] \Delta(\tau) \mathbf{D} \phi \exp \left[-\frac{26-D}{48 \pi} S_{L}[\phi]\right) \\
& \times \delta\left[\int e^{-\phi \sqrt{\hat{g}} d^{2} \xi-A}\right] .
\end{aligned}
$$

The number of random surfaces $F_{\chi}(A)$ is related to the partition function by the expression (1.3). Let $\phi_{A}$ be the classical_minimum of $S_{L}$, under the constraint $\int e^{-\phi} \sqrt{\hat{g}} d^{2} \xi=A$ :

$$
\begin{aligned}
& \delta S_{L}=\int \sqrt{\hat{g}} e^{-\phi} R\left(e^{-\phi} \widehat{g}\right) \delta \phi d^{2} \xi=0, \\
& \int \sqrt{\hat{g}} e^{-\phi} \delta \phi d^{2} \xi=0 .
\end{aligned}
$$

These equations are satisfied if $R\left(e^{-\phi} \hat{g}\right)$ is a constant. From a general theorem on two-dimensional Riemann surfaces, there exists a scalar field $\phi_{A}$, for which $R=R\left(e^{-\phi} \widehat{g}\right)$ is constant and $\phi_{A}$ is unique up to an additive constant. This constant is fixed by the constraint

$$
\int e^{-\phi} \sqrt{\hat{g}} d^{2} \xi=A \text {. }
$$

Let us express

$$
\phi_{A}(\xi)=\phi_{1}(\xi)-\ln A \quad,
$$

where $\phi_{1}(\xi)$ is the solution for $A=1$, and define $g_{1}=e^{-\phi_{1}} \widehat{g}$. Then the classical minimum can be expressed as

$$
\begin{aligned}
S_{L}\left[\phi_{A}(\xi)\right] & =S_{L}\left[\phi_{1}(\xi)\right]+\int d^{2} \xi \sqrt{g_{1}} R\left(g_{1}\right) \ln A \\
& =S_{L}\left[\phi_{1}(\xi)\right]+4 \pi \chi \ln A
\end{aligned}
$$


Thus, at the tree level we have the simple area dependence,

$$
\exp \left[\frac{-(26-D)}{48 \pi} S_{L}\right] \sim A^{[(D-26) / 12] \chi}
$$

Next, let us consider the world-sheet one-loop correction to this behavior from the saddle-point expansion of the Liouville action about its classical value. If we introduce the shifted field $\widetilde{\phi}=\phi-\phi_{A},(2.35)$ can be rewritten as

$$
\begin{aligned}
F_{\chi}(A)=V \int & \mathbf{D} \widetilde{\phi}[d \tau] \Delta(\tau)\left(e^{[-(26-D) / 48 \pi] S_{L}\left[\phi_{1}\right]} A^{[(D-26) / 12] \chi}\right) \\
& \times \exp \left(\frac{-(26-D)}{48 \pi} \int d^{2} \xi\left(\frac{1}{2} \sqrt{g_{A}} g_{A}^{m n} \partial_{m} \widetilde{\phi} \partial_{n} \widetilde{\phi}+\sqrt{g_{A}} R_{A} \widetilde{\phi}\right)\right) \delta\left(\int \sqrt{g_{A}} e^{-\bar{\phi}}-A\right),
\end{aligned}
$$

where $g_{A}=e^{-\phi_{A}} \hat{g}$. At the one-loop level, we can introduce the following truncations:

$$
\|\delta \widetilde{\phi}\|^{2}=\int \sqrt{g_{A}} e^{-\bar{\phi}}(\delta \widetilde{\phi})^{2} d^{2} \xi \cong \int \sqrt{g_{A}}(\delta \widetilde{\phi})^{2} d^{2} \xi
$$

and neglecting terms of $O\left(\widetilde{\phi}^{3}\right)$,

$$
\delta\left[\int \sqrt{g_{A}} e^{-\bar{\phi}} d^{2} \xi-A\right] \cong \delta\left[\int \sqrt{g_{A}}\left(\widetilde{\phi}-\frac{\widetilde{\phi}^{2}}{2}\right] d^{2} \xi\right] .
$$

\section{Using}

$$
\int \sqrt{g_{A}} \widetilde{\phi} d^{2} \xi=\frac{1}{2}\left[\int \sqrt{g_{A}} \widetilde{\phi}^{2} d^{2} \xi\right]+O\left(\widetilde{\phi}^{3}\right),
$$

to replace the linear term in $\widetilde{\phi}$ in the action, gives

$$
\int \sqrt{g_{A}} R\left(g_{A}\right) \widetilde{\phi} d^{2} \xi \sim \frac{1}{2} \int \sqrt{g_{A}} R\left(g_{A}\right) \widetilde{\phi}^{2} d^{2} \xi,
$$

since $R\left(g_{A}\right)$ is a constant given by $4 \pi \chi / A$. Thus the one-loop expression for $F_{\chi}(A)$ is

$$
\begin{aligned}
F_{\chi}(A)_{1 \text { loop }}=A^{[(D-26) / 12] \chi} V \int & {[d \tau] \Delta(\tau) \exp \left(-\frac{26-D}{48 \pi} S_{L}\left[\phi_{1}\right]\right) } \\
& \left.\times \operatorname{det}^{\prime}\left(\Delta_{g_{A}}+\frac{4 \pi \chi}{\int \sqrt{g_{A}} d^{2} \xi}\right)^{-1 / 2} \iint \sqrt{g_{A}} d^{2} \xi\right)^{1 / 2},
\end{aligned}
$$

the last factor coming from the measure, and the constraint for the constant mode of $\widetilde{\phi}$. The determinant can be evaluated as follows:

$$
\operatorname{det}^{\prime}\left(\Delta_{g_{A}}+\frac{4 \pi \chi}{\int \sqrt{g_{A}} d^{2} \xi}\right)^{-1 / 2}=\left(\operatorname{det}^{\prime} \Delta_{g_{A}}\right)^{-1 / 2} \exp \left[-\frac{1}{2} \operatorname{Tr} \ln \left[1+\frac{4 \pi \chi}{\int \sqrt{g_{A}} d^{2} \xi} \Delta_{g_{A}}^{-1}\right)\right] .
$$

From Eq. (2.39), the first factor can be rewritten as

$$
\left(\operatorname{det}^{\prime} \Delta_{g_{A}}\right)^{-1 / 2}\left[\int \sqrt{g_{A}} d^{2} \xi\right]^{1 / 2}=\left(\operatorname{det}^{\prime} \Delta_{g_{1}}\right)^{-1 / 2}\left[\int \sqrt{g_{1}} d^{2} \xi\right]^{1 / 2} A^{\chi / 12} .
$$

The second factor in (2.47) may be expressed as a Taylor expansion:

$$
\operatorname{Tr} \ln \left(1+\frac{4 \pi \chi}{\int \sqrt{g_{A}} d^{2} \xi} \Delta_{g_{A}}^{-1}\right]=\frac{4 \pi \chi}{A} \operatorname{Tr} \Delta_{g_{A}}^{-1}-\frac{1}{2}\left[\left(\frac{4 \pi \chi}{A}\right)^{2} \operatorname{Tr}\left(\Delta_{g_{A}}\right)^{-2}\right]+\cdots .
$$

The first term in (2.49) contributes a $\ln A$ dependence, while the higher-order terms are free of $\ln A$ corrections:

$$
\begin{aligned}
& \frac{1}{A} \operatorname{Tr} \Delta_{g_{A}}^{-1} \cong \frac{1}{A} \sum_{k_{i}=0}^{\Lambda A} \frac{\left(\Delta k_{i}\right)^{2}}{(2 \pi)^{2}} \frac{1}{-k_{i}^{2} / A} \cong \frac{-1}{4 \pi} \ln A+\text { const }, \\
& \frac{1}{A^{2}} \operatorname{Tr} \Delta_{g_{A}}^{-2} \cong \frac{1}{A^{2}} \sum_{k_{i}=0}^{\Lambda A} \frac{\left(\Delta k_{i}\right)^{2}}{(2 \pi)^{2}}\left(\frac{1}{-k_{i}^{2} / A}\right)^{2} \cong \text { const } \times O\left(A^{0}\right) .
\end{aligned}
$$

Thus, 


$$
\operatorname{Tr} \ln \left[1+\frac{4 \pi \chi}{\int \sqrt{g_{A}} d^{2} \xi} \Delta_{g_{A}}^{-1}\right]=K A-\chi \ln A+B(\tau)+O(1 / A),
$$

where $K$ is a regularization-dependent constant. The final expression for $F_{\chi}(A)$ is

$$
F_{\chi}(A) \underset{A \rightarrow \infty}{\sim} C_{\chi} A^{[(D-19) / 12] \chi-1} e^{K A},
$$

where

$$
C_{\chi}=V \int \frac{[d \tau]}{\mathbf{\Omega}_{\hat{g}}\left(\left\{\mathbf{K}_{\hat{g}}\right\}\right)}\left(\frac{\operatorname{det}^{\prime} \Delta_{\hat{g}}}{\int \sqrt{\hat{g}} d^{2} \xi}\right)^{-D / 2}\left(\operatorname{det}^{\prime} \hat{P}^{\dagger} \hat{P}\right)^{1 / 2} e^{-[(26-D) / 48 \pi] S_{L}\left[\phi_{1}\right]}\left(\frac{\operatorname{det}^{\prime} \Delta_{g_{1}}}{\int \sqrt{g_{1}} d^{2} \xi}\right)^{1 / 2} e^{B(\tau)},
$$

and the critical exponent $b_{\chi}=(D-19) \chi / 12-1$.

Of course, this is only the one-loop result obtained by expanding the Liouville action about its classical value. In practice, we expect this result to be exact. As discussed in Ref. 17 for the case of a spherical topology $(\chi=2)$ higher-loop contributions do not alter the leading area dependence.

In the critical dimension, $D=26$, this analysis becomes meaningless because of conformal invariance. In this case, $(2.10)$ is replaced by

$Z_{(D=26)}=\int \mathbf{D} X \frac{\mathbf{D} g}{\Omega_{g}(\{\mathbf{C}\}) \Omega_{g}(\{\mathbf{D}\})} \exp (-S)$,

where $\Omega_{g}(\{\mathrm{C}\})$ is the conformal volume. It is easy to divide by this new factor; the net effect is equivalent to setting the $\phi$ field to some arbitrary value. Thus,

$$
\begin{gathered}
\left.Z_{(D=26)=V \int[}=\frac{d \tau]\left[\operatorname{det}\left(\hat{P}^{\dagger} \hat{P}\right)\right]^{1 / 2}}{\int \sqrt{\hat{g}} d^{2} \xi}\right]^{-13} .
\end{gathered}
$$

\section{FERMIONIC STRINGS AND SPIN STRUCTURES}

In our discussion this far, we have only considered bosonic variables on the world sheet. In order to place fermions on the world sheet, we must introduce the zweibein field $e_{m}^{a}$ on the manifold. Corresponding to any Riemannian metric $g_{m n}$, there exists a set of zweibeins satisfying

$$
g_{m n}(\xi)=e_{m}^{a} e_{n}^{b} \delta_{a b} .
$$

In general, zweibeins cannot be defined globally; one must introduce a set of patches that cover the manifold. The zweibeins are then defined on each patch $U$ and there are transition functions relating the zweibeins on every pair of overlapping patches:

$e_{m}^{(i) a}(\xi) d \xi^{m}=L_{i j}(\xi)_{b}^{a} e_{m}^{(j) b}\left(\xi^{\prime}\right) d \xi_{m}{ }^{\prime}, U \cap U^{\prime} \neq \varnothing$.

$L_{i j b}^{a}$ is the transition function from $U_{i}$ to $U_{j}$ given by $L_{i j b}^{a}=\left(e^{\phi_{i j}(\xi)} \epsilon\right)^{a}{ }_{b}, \epsilon_{b}^{a}$ is the rank-2 antisymmetric matrix, and we have assumed for simplicity that the overlapping region is simply connected. Fermion fields can now be introduced by demanding that the transition functions for the fermions be consistent with those for the zweibeins. However, there is an ambiguity in sign since the spinor representation doubly covers the vector representation. Explicitly, define a spinor field $\psi_{(j)}^{\alpha}$ on each patch $U_{j}$ that satisfies the following transition condition:

$\psi_{(i)}^{\alpha}=\sigma_{i j}\left(e^{\left[i \gamma^{5} \phi_{i j}(\xi)\right] / 2}\right)_{\beta}^{\alpha} \psi_{(j)}^{\beta}$ if $U_{i} \cap U_{j} \neq \varnothing$,

where $\sigma_{i j}= \pm 1$, and $\phi_{i j}(\xi)$ is fixed so that the choice $\sigma_{i j}=+1$ gives a consistent spin structure. Then $\sigma_{i j}$ must satisfy the cocycle condition in the triply overlapped region:

$$
\sigma_{i j} \sigma_{j k}=\sigma_{i k}, \quad U_{i} \cap U_{j} \cap U_{k} \neq \varnothing .
$$

However, since the Lagrangian contains only even powers of the spinor fields, it is invariant under the change

$$
\psi_{i}^{\alpha}(\xi) \rightarrow-\psi_{i}^{\alpha}(\xi) \quad,
$$

on the patch $U_{i}$. This means that spin bundles consistent with the original zweibein structure are classified by the one-dimensional cohomology group with $Z_{2}$ coefficients, $H^{1}\left(M ; Z_{2}\right)$.

For example, a sphere has only one class of spin bundles, while a torus has four classes of spin bundles (four spin structures) since $H^{1}$ (torus; $Z_{2}$ ) $=Z_{2} \otimes Z_{2}$. This can be seen more simply as follows: one can regard a torus as a parallelogram (Fig. 3) with periodic boundary conditions on which the zweibeins also satisfy periodicity. The zweibein can be rotated by a phase angle $2 \pi n$ each time it is transported around some noncontractible loop on the torus. The integer $n$ is unchanged by local Lorentz transformations and can only be changed by a global Lorentz transformation, or alternatively a modular transformation. Since spinor fields can be either periodic or antiperiodic in the $\left(\xi_{1}, \xi_{2}\right)$ directions, there are four spin structures. In general, for a Riemann surface with $h$ handles, there are $4^{h}$ spin structures.

Chiral spin bundles can be analyzed in a similar manner. In this case, there are ambiguities in sign for each chirality separately. However, when we introduce left-right asymmetry into the model, the phase of the path integral becomes ill defined, and as mentioned before, there is the possibility that a gravitational anomaly can arise.

Next, consider the possibility of putting an arbitrary two-dimensional conformal field theory on the world 


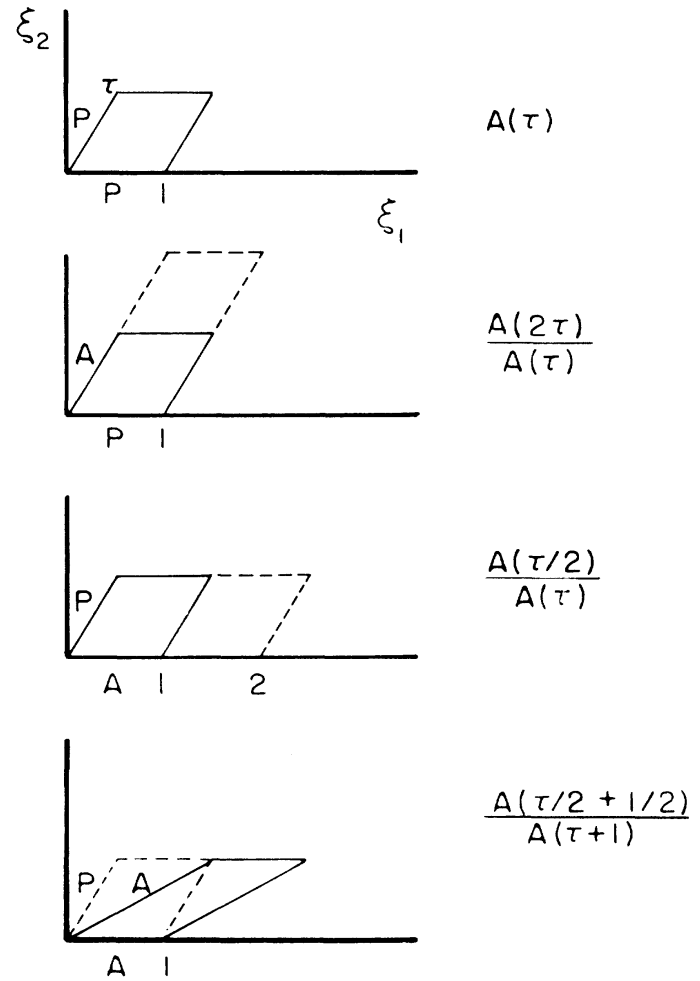

FIG. 3. The evaluation of the determinants with different boundary conditions on the torus. (a) The standard periodicperiodic $(P P)$ case gives the function $A(\tau)$ which is modular invariant except for an overall phase; (b) the antiperiodicperiodic $A P$ case; (c) the $P A$ case where the enlarged domain shown must be rescaled so that the $\xi_{1}$ period is of unit length; and (d) the $A A$ case is related to the $P A$ case as shown.

sheet. Fields with fractional spin other than half-integer value can only be introduced on manifolds with certain special values of the Euler characteristic $\chi$. This is easily demonstrated: let us transport the field with spin $s$ along a loop $C$, that cuts the manifold $M$ into two parts $M=M_{1} \cup M_{2}$. Since the loop $C$ can be regarded as the boundary of either $M_{1}$ or $M_{2}$ while the resulting phase change should be independent of this choice, from the Gauss-Bonnet theorem, we have the condition

$$
4 \pi \chi s=2 \pi n,
$$

and hence the requirement that $2 \mathrm{~s} \chi$ must be integer valued. For example, a spin- $\frac{1}{3}$ field cannot be put on a manifold topologically equivalent to the sphere $(\chi=2)$ (an exception occurs when there is a monopolelike object inside the sphere $S^{2}$ ).

The conformal anomaly for a left-right-symmetric conformal field theory on the world sheet is given by the sum of the central charges of the matter fields, minus $26 \mathrm{com}$ ing from the Faddeev-Popov determinant. For such a model the critical exponent is given by the general expression

$$
b=\left(\sum_{i} c_{i}-19\right) \frac{\chi}{12}-1,
$$

where the $c_{i}$ 's are the central charges of the fields introduced on the world sheet. If we do not maintain leftright symmetry, we must check the anomaly cancellation conditions. For the well-known gravitational anomaly associated with $\left\{D_{0}\right\}$, this condition is ${ }^{19}$

$$
\left(\sum_{i=1}^{n} c_{i}-26\right)-\left(\sum_{i=1}^{n} \bar{c}_{i}-26\right)=0,
$$

where $c_{i}$, and $\bar{c}_{i}$ are the central charges for left-moving and right-moving fields, respectively. This is easy to see: the gravitational anomaly can be viewed as a local Lorentz transformation and hence, as a conformal transformation with a pure imaginary parameter that enters with opposite sign for left- and right-moving fields. The cancellation condition for the modular anomaly gives another set of constraints, which are discussed in Sec. VI.

\section{THE COVARIANT PATH INTEGRAL FOR THE NSR SUPERSTRING}

We extend the preceding discussion to the path-integral formulation of the Neveu-Schwarz-Ramond (NSR) model, first discussed by Polyakov. ${ }^{7}$ Our starting point is the two-dimensional $(N=1)$ supergravity theory. It is convenient to construct the functional integral for the NSR string in superspace, maintaining manifest invariance under both diffeomorphisms and local supersymmetry transformations.

The superfield formulation of the fermionic string action was carried out by Howe, ${ }^{15}$ and unless stated otherwise, we follow his notation. The partition function was first discussed by Martinec, ${ }^{8}$ who also generalized the heat-kernel method and the complex tensor calculus to the fermionic string. An advantage of this approach over the Wess-Zumino gauge-fixed approach is that the superspace formulation can be discussed in a way closely parallel to that of the bosonic string. Also, lengthy and often unnecessary calculations can be avoided.

For a fixed topology of the base manifold $M$, the partition function of the supersymmetric string is

$$
\begin{aligned}
& Z_{\chi}=\sum_{s} \int \mathrm{D} E_{M}^{A} \mathrm{D} X^{I} \exp (-S[X, E]), \\
& S=\frac{1}{2} \int_{M_{s}} d^{2} z E D_{\alpha} X^{I} D^{\alpha} X^{I},
\end{aligned}
$$

where $z=\left(\xi^{m}, \theta^{\mu}\right)$ are the $(2+2)$ coordinates of the curved supermanifold $M_{s}$, which is constructed as a spin bundle with spin structure $s$ on the base manifold $M . \xi^{m}$ are the coordinates of the two-dimensional world sheet, $\theta^{\mu}$ are the anticommuting coordinates of the fiber, and $d^{2} z=d^{2} \xi d^{2} \theta$.

Here $X^{I}(I=1, \ldots, D)$ is the scalar superfield and $E_{M}^{A}$ the supervierbein, a $4 \times 4$ graded, invertible matrix with superdeterminant $E$. Following Howe, ${ }^{15}$ the coordinate indices are given by letters from the middle of the alphabet, while tangent space indices are taken from the beginning. Small latin (greek) letters stand for bosonic (fermionic) components while, capital letters span both types. 
The covariant derivative is defined by

$$
D_{A}=E_{A}^{M} D_{M}=E_{A}^{M} \partial_{M}+\Omega_{A} L,
$$

where $\Omega_{A}$ is the spin connection and $L$ the Lorentz generator.

The variables in the superfield formulation of supergravity are highly redundant. This redundancy can be removed by imposing kinematic constraints on some of the components of the supertorsion tensor, $T_{A B}^{C}$, defined by

$$
\left[D_{A}, D_{B}\right\}=T_{A B}^{C} D_{C}+R_{A B} L \text {, }
$$

where $R_{A B}$ is the curvature tensor. The kinematic constraints are given by

$$
\begin{aligned}
& T_{\alpha \beta}{ }^{\gamma}=0, \\
& T_{\alpha \beta}^{a}=2 i\left(\gamma^{a}\right)_{\alpha \beta}, \\
& T_{a b}{ }^{c}=0 .
\end{aligned}
$$

Using the Bianchi identity $R_{[\alpha \beta \gamma]}{ }^{d}=0$, we find $T_{\beta c}^{a}=0$. As a consequence of these constraints, the volume element $\mathbf{D} E_{M}^{A}$ needs careful treatment.

$E_{A}^{M}$ has 16 superfields, and there are a total of $(6+6+2=14)$ constraints in (4.5). Four of them determine $\Omega_{A}$ in terms of the supervierbeins. The rest can be used to express $E_{a}^{M}$ in terms of $E_{\alpha}^{M}$ and, in addition, set two constraints on $E_{\alpha}^{M}$, leaving only six independent superfields in $E_{A}^{M}$. However, local Lorentz invariance and superdiffeomorphisms remove $(1+4=5)$ degrees of freedom so that there is actually only one independent superfield. In the presence of superconformal invariance, this remaining degree of freedom will be removed by a superconformal transformation.

It is convenient to express $\delta E_{A}^{M}$ in terms of the following six independent superfields that span the variations $\delta E_{M}^{B}$ :

$$
\delta H_{b}^{\alpha}, \quad\left(\gamma^{5}\right)_{\alpha}^{\beta} \delta H_{\beta}^{\alpha}, \quad \delta H_{\alpha}^{\alpha},
$$

with $\delta H_{A}^{B} \equiv\left(\delta E_{A}^{M}\right) E_{M}^{B}$. Then the functional volume element $\mathbf{D} E_{A}^{M}$ is defined by the metric ${ }^{8}$

$$
\begin{gathered}
\|\delta E\|^{2}=\int d^{2} z E\left[\left(\delta H_{b}^{\alpha}\right)\left(\delta H_{\alpha}^{b}\right)+c_{1}\left(\gamma_{\alpha}^{5 \beta} \delta H_{\beta}^{\alpha}\right)^{2}\right. \\
\left.+c_{2}\left(\delta H_{\alpha}^{\alpha}\right)^{2}\right],
\end{gathered}
$$

where $c_{1}$ and $c_{2}$ are arbitrary coefficients. It should be noted that this definition of norm is for a nonchiral sum over spin structures of the amplitudes in Eq. (4.1). (See note added.)

We point out a potential difficulty here. The supervierbein functional space appears to have indefinite norm. However, by a judicious choice of gauge slice this difficulty can be overcome. For example, one can set the arbitrary constants equal to zero, and choose the fiducial gauge slice transverse to the action of the super-Weyl group to be the space of vierbeins having constant supercurvature. Small variations away from the fiducial supervierbein have the typical form (in a complex tensor basis)

$$
\begin{aligned}
& \delta H_{+}^{z}=D_{+} \delta H_{+}^{-}+R_{+-} \delta H_{\bar{z}}^{-}+O\left(\delta H^{2}\right), \\
& \delta H_{-}^{z}=D_{-} \delta H_{+}^{-}+R_{-+} \delta H_{z}^{+}+O\left(\delta H^{2}\right) .
\end{aligned}
$$

Hence, the metric defined in (4.7) can be made positive definite for genus $h>1$. The proper treatment of the norm for genus 1 remains an open issue. (This issue enters only in the case of periodic boundary conditions for the fermions in scattering amplitudes. For the vacuum amplitude, this problem does not arise since supermoduli are absent for the three contributing choices of boundary condition.)

The functional volume element $\mathbf{D} X$ is a straightforward generalization of the bosonic case:

$$
\|\delta X\|^{2}=\int d^{2} z E(\delta X)^{2} .
$$

The action and the functional volume elements, $\mathbf{D} X$ and $\mathbf{D} E_{M}^{A}$, are invariant under the group of superdiffeomorphisms $\{\mathrm{SD}\}$ and under local Lorentz transformations $\{\mathbf{L}\}$. A superdiffeomorphism is a differentiable supermapping of $M_{s}$ onto itself,

$$
z \in M_{s} \rightarrow z^{\prime}(z) \in M_{s}
$$

under which $X^{I}$ and $E_{M}^{A}$ transform as

$$
\begin{aligned}
& X^{I}(z) \rightarrow X^{I^{\prime}}(z), \quad X^{I^{\prime}}\left(z^{\prime}\right)=X^{I}(z), \\
& E_{A}^{M}(z) \rightarrow E_{A}^{M^{\prime}}(z), \quad E_{A}^{M^{\prime}}\left(z^{\prime}\right) \partial_{M}^{\prime}=E_{A}^{M}(z) \partial_{M} .
\end{aligned}
$$

Under a local Lorentz transformation, the superfields transform as follows:

$$
\begin{aligned}
& X^{I}(z) \rightarrow X^{I}(z) \quad(\text { no change) } \\
& E_{M}^{\alpha}(z) \rightarrow\left[\exp \left[\frac{i \gamma^{5}}{2} \Phi(z)\right]\right]_{\beta}^{\alpha} E_{M}^{\beta}(z), \\
& E_{M}^{a}(z) \rightarrow\{\exp [\epsilon \Phi(z)]\}_{b}^{a} E_{M}^{b}(z),
\end{aligned}
$$

where $\epsilon$ is given by $\epsilon_{a b}=-\epsilon_{b a}$ and $\epsilon_{12} \equiv 1$. The action is also invariant under the group of local superconformal (SC) transformations:

$$
\begin{aligned}
& X^{I} \rightarrow X^{I} \quad \text { (no change) } \\
& E_{\alpha}^{M} \rightarrow \Lambda^{-1 / 2} E_{\alpha}^{M} \\
& E_{a}^{M} \rightarrow \Lambda^{-1} E_{a}^{M}+i \Lambda^{-2}\left(\gamma_{a}\right)^{\alpha \beta}\left(D_{\beta} \Lambda\right) E_{\alpha}^{M} .
\end{aligned}
$$

We shall denote the group of superconformal transformations by $\{\mathrm{SC}\}$. As in the bosonic case, the metric in (4.7) and (4.8) is not invariant under superconformal transformations, giving rise to the superconformal anomaly. To separate possible global anomalies from the supergravitational anomaly, it is convenient to factor out $\left\{\mathrm{SD}_{0}\right\}$, the set of superdiffeomorphisms continuously connected to the identity, from $\{S D\}$. We define the super mappingclass group $G_{s}$ as the factor group

$$
G_{s}=\{\mathrm{SD}\} /\left\{\mathrm{SD}_{0}\right\}
$$

with order $\left|G_{s}\right|$. Intuitively, one might expect that fermionic coordinates $\theta^{\mu}$ do not affect the global structure of the space of diffeomorphisms, so that $G_{s}=G$ $\equiv\{\mathrm{D}\} /\left\{\mathbf{D}_{0}\right\}$.

Taking the superdiffeomorphism and local Lorentz invariance into account, we replace the functional measure in (4.1) by 


$$
\int \mathrm{D} E_{M}^{A} \mathbf{D} X^{I} \rightarrow \int \frac{\mathbf{D} E_{M}^{A} \mathbf{D} X^{I}}{\left|G_{s}\right| \mathbf{\Omega}_{E}\left(\{\mathrm{~L}\}\left(x\left\{\mathrm{SD}_{0}\right\}\right)\right.},
$$

where $\boldsymbol{\Omega}_{E}\left(\{\mathbf{L}\} \otimes\left\{\mathbf{S D}_{0}\right\}\right)$ is the volume of the semidirect product of the gauge groups $\left\{\mathrm{SD}_{0}\right\}$ and $\{\mathrm{L}\}$. Let us introduce a gauge slice $S_{s}^{\prime}$, that represents $\mathbf{m}_{s}^{\prime} \equiv \mathbf{m}_{s} /$ $\{L\} \otimes\left\{\mathbf{S D}_{0}\right\}$, where $\mathbf{m}_{s}$ is the space of all supervierbeins on $M_{s}$ satisfying the kinematic constraints (4.5). The space

$$
\mathbf{m}_{s}^{\prime} /\{\mathbf{S C}\} \otimes\{\mathbf{L}\} \otimes\left\{\mathbf{S D}_{0}\right\}
$$

which may be called super-Teichmueller space, is a finitedimensional supermanifold. Fixing a representation $S_{s}=\left\{\widehat{E}_{A}^{M}(z ; T)\right\}$ of super-Teichmueller space, an element of the gauge slice $S_{s}^{\prime}=\left\{E_{A}^{M}(z)\right\}$ can be expressed as

$$
\begin{aligned}
E_{\alpha}^{M}(z)= & e^{[\Phi(z)] / 2} \hat{E}_{\alpha}^{M}(z ; T), \\
E_{a}^{M}(z)= & e^{\Phi(z)} \hat{E}_{a}^{M}(z ; T) \\
& -i e^{\Phi(z)}\left(\gamma_{a}\right)^{\alpha \beta}\left[D_{\beta} \Phi(z)\right] \hat{E}_{\alpha}^{M}(z ; T),
\end{aligned}
$$

where the $T$ 's are both bosonic and fermionic $c$ numbers that parametrize super-Teichmueller space. $\Phi$ is the superconformal superfield. An arbitrary infinitesimal variation $\delta E_{A}^{M} \in T_{\mathbf{m}_{s}^{\prime}}$ in the tangent space of $\mathbf{m}_{s}^{\prime}$ at some $E_{A}^{M} \in S^{\prime}$ can be expressed as

$$
\delta E_{A}^{M}=E_{B}^{M} \delta H_{A}^{B},
$$

where $\delta H_{A}^{B}$ is given by

$$
\begin{aligned}
\delta H_{A}^{B}= & \delta Q^{C} T_{C A}^{B}-D_{A} \delta Q^{B}+\left(\delta Q^{C} \Omega_{C}+\delta K\right) E_{A}^{B} \\
& +\delta \Phi \Delta_{A}^{B}+\delta T_{i} \frac{\partial E_{A}^{M}}{\partial T_{i}} E_{M}^{B}
\end{aligned}
$$

$E_{A}^{B}$ and $\Delta_{A}^{B}$ are, respectively,

$$
E_{a}^{b}=\epsilon_{a}^{b}, \quad E_{a}^{\beta}=E_{\alpha}^{a}=0, \quad E_{\alpha}^{\beta}=\frac{1}{2}\left(i \gamma_{5}\right)_{\alpha}^{\beta},
$$

and

$$
\Delta_{a}^{b}=\delta_{a}^{b}, \Delta_{a}^{\beta}=\Delta_{\alpha}^{b}=0, \Delta_{\alpha}^{\beta}=\frac{1}{2}\left(\delta_{\alpha}^{\beta}\right) .
$$

$\delta Q^{A}, \delta \Phi$, and $\delta K$ are, respectively, infinitesimal superdiffeomorphisms, superconformal transformations, and local Lorentz transformations. With the help of the kinematic constraints, the independent components of (4.17) can be obtained:

$$
\begin{aligned}
& \delta H_{\alpha}^{\alpha}=\delta \Phi-D_{\alpha} \delta Q^{\alpha}+\delta T_{i} \frac{\partial \widehat{E}_{\alpha}^{M}}{\partial T_{i}} \widehat{E}_{M}^{\alpha}, \\
& \delta H_{\alpha}^{b}=2 i \delta Q^{\beta}\left(\gamma^{b}\right)_{\beta \alpha}-D_{\alpha} \delta Q^{b}+\delta T_{i} e^{-\Phi / 2} \frac{\partial \hat{E}_{\alpha}^{M}}{\partial T_{i}} \widehat{E}_{M}^{b},
\end{aligned}
$$

$$
\begin{aligned}
\left(\gamma^{5}\right)_{\beta}^{\alpha} \delta H_{\alpha}^{\beta}= & \delta K+\delta Q^{c} \Omega_{c}-\left(\gamma^{5}\right)_{\beta}^{\alpha} D_{\alpha} \delta Q^{\beta} \\
& +\delta T_{i}\left(\gamma^{5}\right)_{\beta}^{\alpha} \frac{\partial \hat{E}_{\alpha}^{M}}{\partial T_{i}} \hat{E}_{M}^{\beta}
\end{aligned}
$$

Now $\delta H_{\alpha}^{b}$ can be decomposed into spin- $\frac{3}{2}$ and spin- $\frac{1}{2}$ components:

$$
\delta H_{\alpha}^{b}=\left[\delta H\left(\frac{1}{2}\right)\right]_{\alpha}^{b}+\left[\delta H\left(\frac{3}{2}\right)\right]_{\alpha}^{b},
$$

where

$$
\begin{aligned}
{\left[\delta H\left(\frac{3}{2}\right)\right]_{\alpha}^{b}=} & \frac{1}{2}\left[\delta H_{\beta}^{c}\left(\gamma^{b} \gamma_{c}\right)_{\alpha}^{\beta}\right] \\
= & -\frac{1}{2}\left(D_{\beta} \delta Q^{c}\right)\left(\gamma^{b} \gamma_{c}\right)_{\alpha}^{\beta} \\
& +\frac{1}{2}\left(\delta T_{i} e^{-\Phi / 2} \frac{\partial \hat{E}_{\beta}^{M}}{\partial T_{i}} \hat{E}_{M}^{c}\left(\gamma^{b} \gamma_{c}\right)_{\alpha}^{\beta}\right)
\end{aligned}
$$

and

$$
\left[\delta H\left(\frac{1}{2}\right)\right]_{\alpha}^{b}=\frac{1}{2}\left[\delta H_{\beta}^{c}\left(\gamma_{c} \gamma^{b}\right)_{\alpha}^{\beta}\right]
$$

With this decomposition,

$$
\left(\begin{array}{c}
\delta H_{\alpha}^{\alpha} \\
\left(\gamma^{5}\right)_{\beta}^{\alpha} \delta H_{\alpha}^{\beta} \\
{\left[\delta H\left(\frac{1}{2}\right)\right]_{\alpha}^{b}} \\
{\left[\delta H\left(\frac{3}{2}\right)\right]_{\alpha}^{b}} \\
\delta C_{i}
\end{array} \mid=\left(\begin{array}{ccccc}
1 & * & * & * & * \\
0 & 1 & * & * & * \\
0 & 0 & \left(\gamma^{b}\right)_{\beta \alpha} & * & * \\
0 & 0 & 0 & -P & * \\
0 & 0 & 0 & 0 & J^{i j}
\end{array}\right)\left(\begin{array}{c}
\delta \Phi \\
\delta K \\
\delta Q^{\beta} \\
\delta Q^{a} \\
\delta T_{j}
\end{array}\right)\right.
$$

Here, $P$ is the differential operator

$$
P: V^{(1)} \rightarrow V^{(3 / 2)},
$$

where $V^{(1)}$ is the space of Lorentz vector superfields, and $V^{(3 / 2)}$ is the space of Lorentz spin- $\frac{3}{2}$ superfields. Explicitly,

$$
(P \delta Q)_{\alpha}^{b}=\frac{1}{2}\left(D_{\beta} \delta Q^{c}\right)\left(\gamma^{b} \gamma_{c}\right)_{\alpha}^{\beta} .
$$

As for the bosonic string, zero modes of $P$ must be excluded from $\delta Q$ in order to avoid redundancy, so that

$$
\delta Q \in(\operatorname{ker} P)^{\perp},
$$

where $\operatorname{ker} P$ is the space of superconformal Killing vectors. A basis for $\operatorname{ker} P^{\dagger},\{\Psi(i)\}$, is defined by the relation

$$
\left\langle\Psi^{(i)} \mid \Psi^{(l)}\right\rangle_{E} J_{l}^{j} \equiv\left\langle\Psi^{(i)} \mid \frac{\partial E_{\beta}^{M}}{\partial T_{j}} E_{M}^{a}\right\rangle_{E},
$$

where the inner product must be defined by the metric (4.7), and the relationship between $E_{M}^{A}$ and $\widehat{E}_{M}^{A}$ is as given by (4.16).

Extending the argument given in the bosonic construction we introduce the superdimension of superTeichmueller space, equal to the super-dimension of $\operatorname{ker} P^{\dagger}$,

$$
\operatorname{sdim}\left(S_{s}\right)=\operatorname{sdim}\left(\operatorname{ker} P^{\dagger}\right),
$$

and define it as follows: the superdimension is the pair of numbers $(b, f)$, where $b$ is the bosonic dimension, or the number of moduli, and $f$ is the fermionic dimension, or the number of supermoduli. For example, if the superspace is a trivial spin bundle on a torus,

$$
\begin{aligned}
& \operatorname{sdim}(\operatorname{ker} P)=(2,2), \\
& \operatorname{sdim}\left(\operatorname{ker} P^{\dagger}\right)=(2,2) .
\end{aligned}
$$


However, if the superspace is a nontrivial bundle, for which there are three possibilities on the torus,

$$
\begin{aligned}
& \operatorname{sdim}(\operatorname{ker} P)=(2,0), \\
& \operatorname{sdim}\left(\operatorname{ker} P^{\dagger}\right)=(2,0) .
\end{aligned}
$$

The sphere has $\operatorname{sdim}(\operatorname{ker} P)=(6,4), \quad \operatorname{sdim}\left(\operatorname{ker} P^{\dagger}\right)=(0,0)$. For Riemannian surfaces with genus $h>1$, the RiemannRoch theorem gives

$$
\begin{aligned}
& \operatorname{sdim}(\operatorname{ker} P)=(0,0) \\
& \operatorname{sdim}\left(\operatorname{ker} P^{\dagger}\right)=(6(h-1), 4(h-1))
\end{aligned}
$$

These results may be easily confirmed by going to the Wess-Zumino gauge.

The calculation of the functional measure in (4.15) is a straightforward generalization of the bosonic case:

$$
\int \frac{\mathbf{D} E}{\Omega_{E}\left(\{\mathrm{~L}\} \times\left\{\mathrm{SD}_{0}\right\}\right)}=\int \frac{\mathbf{D} \Phi\left[\operatorname{sdet}^{\prime}\left(P^{\dagger} P\right)\right]^{1 / 2} \operatorname{sdet}\left\langle\Psi^{(i)} \mid \frac{\partial \hat{E}_{\beta}^{M}}{\partial T_{j}} \hat{E}_{M}^{a}\right\rangle_{E}}{\Omega_{E}(\{\mathrm{SCK}\})\left(\operatorname{sdet}\left\langle\Psi^{(i)} \mid \Psi^{(j)}\right\rangle_{E}\right)^{1 / 2}} \prod_{i} d T_{i} .
$$

The measure for $\mathrm{D} \Phi$ is induced from the third term of (4.7) and is given by the metric

$$
\|\delta \Phi\|^{2}=\int E(\delta \Phi)^{2} d^{2} z=\int e^{-\Phi} \hat{E}(\delta \Phi)^{2} d^{2} z
$$

$\Omega_{E}(\{\mathrm{SCK}\})$ denotes the volume of the space spanned by the superconformal Killing vectors.

Using the heat-kernel method in superspace, the $\Phi$ dependence of the integrand in (4.31) can be calculated. Let us introduce the vacuum functional

$$
W\left[E_{\alpha}^{M}\right]=\ln \left(\frac{\left[\operatorname{sdet}^{\prime}\left(P^{\dagger} P\right)_{E}\right]^{1 / 2} \operatorname{sdet}\left\langle\Psi^{(i)} \mid \frac{\partial \hat{E}_{\beta}^{M}}{\partial T_{i}} \hat{E}_{M}^{a}\right\rangle_{E}}{\Omega_{E}(\{\mathrm{SCK}\})\left(\operatorname{sdet}\left\langle\Psi^{(i)} \mid \Psi^{(j)}\right\rangle_{E}\right)^{1 / 2}}\right) .
$$

Then

$$
\delta W\left[E_{\alpha}^{M}\right]=W\left[e^{\delta \Phi(z) / 2} E_{\alpha}^{M}\right]-W\left[E_{\alpha}^{M}\right]=\frac{c_{\mathrm{FP}}}{48 \pi} \int E Y \delta \Phi d^{2} z
$$

where $c_{\mathrm{FP}}=-(26-11)=-15$ is the central charge of the super-Virasoro algebra of the supersymmetric Faddeev-Popov ghost field, and $Y$ is the curvature superfield defined by

$$
\left(\gamma_{5}\right)_{\alpha \beta} Y=R_{\alpha \beta} \text {. }
$$

Recall that the kinematic constraints leave six independent superfields in $E_{A}^{M}$. Using the Bianchi identitites and the kinematic constraints as described in Appendix B, all components of the Riemann curvature tensor $R_{A B}$ and the supertorsion $T_{A}^{B C}$ can be expressed as functions of $Y$ (Ref. 15).

Following similar arguments to those given in the bosonic construction, we define

$$
Y\left(e^{\Phi / 2} E_{\alpha}^{M}\right)=e^{\Phi}\left(Y-D_{\alpha} D^{\alpha} \Phi\right)
$$

Integrating (4.33) gives

$$
W\left[e^{\Phi / 2} \hat{E}_{\alpha}^{M}\right]-W\left[\hat{E}_{\alpha}^{M}\right]=\frac{-15}{48 \pi} \int \hat{E}\left(\hat{Y} \Phi+\frac{1}{2} \hat{D}_{\alpha} \Phi \hat{D}^{\alpha} \Phi\right) d^{2} z
$$

where $\hat{Y}$ and $\hat{D}_{\alpha}$ are evaluated at the fiducial reference supervierbein $\hat{E}_{M}^{A}$. Thus,

$$
\frac{\left[\operatorname{sdet}^{\prime}\left(P^{\dagger} P\right)_{E}\right]^{1 / 2} \operatorname{sdet}\left\langle\Psi^{(i)} \mid \frac{\partial \hat{E}_{\beta}^{M}}{\partial T_{i}} \hat{E}_{M}^{a}\right\rangle_{E}}{\Omega_{E}(\{\mathrm{SCK}\})\left(\operatorname{sdet}\left\langle\Psi^{(i)} \mid \Psi^{(j)}\right\rangle_{E}\right)^{1 / 2}}=\frac{\left[\operatorname{sdet}^{\prime}\left(\hat{P}^{\dagger} \hat{P}\right)_{\hat{E}}\right]^{1 / 2} \operatorname{sdet}\left\langle\Psi^{(i)} \mid \frac{\partial \hat{E}_{\beta}^{M}}{\partial T_{i}} \hat{E}_{M}^{a}\right\rangle_{\hat{E}}}{\Omega_{\hat{E}}(\{\mathrm{SCK}\})\left(\operatorname{sdet}\left\langle\Psi^{(i)} \mid \Psi^{(j)}\right\rangle_{\hat{E}}\right)^{1 / 2}} e^{(-15 / 48 \pi) S_{\mathrm{SL}}[\Phi, \hat{E}]}
$$

and the super-Liouville action is given by

$$
S_{\mathrm{SL}}[\Phi, \hat{E}]=\int \hat{E}\left(\hat{Y} \Phi+\frac{1}{2} \widehat{D}_{\alpha} \Phi \widehat{D}^{\alpha} \Phi\right) d^{2} z
$$

Comparing this with (4.2) and (4.34), we see that $S_{\mathrm{SL}}$ is both Lorentz and superdiffeomorphism invariant.

The $X$ integration gives

$$
\int \mathbf{D} X \exp (-S)=V\left(\frac{\operatorname{sdet}^{\prime}\left(D_{\alpha} D^{\alpha}\right)}{\int E d^{2} z}\right)^{-D / 2},
$$

where the volume factor $V$ comes from the constant modes of the superfield. Note that $\operatorname{sdet}^{\prime}\left(D_{\alpha} D^{\alpha}\right)^{-1}$ may still have factors of zero coming from fermionic zero 
modes. For example, as is discussed in Sec. VI, a torus with trivial spin structure has $\operatorname{sdet}^{\prime}\left(D_{\alpha} D^{\alpha}\right)^{-1 / 2}=0$ because of the fermionic zero modes. Using the super heatkernel method, ${ }^{8}$ we have

$$
\begin{aligned}
{\left[\frac{\operatorname{sdet}^{\prime}\left(D_{\alpha} D^{\alpha}\right)_{E}}{\int E d^{2} z}\right]^{-1 / 2}=} & {\left[\frac{\operatorname{sdet}^{\prime}\left(\hat{D}_{\alpha} \hat{D}^{\alpha}\right)_{\hat{E}}}{\int \hat{E} d^{2} z}\right]^{-1 / 2} } \\
& \times \exp \left[\frac{c_{x}}{48 \pi} S_{\mathrm{SL}}[\Phi, \hat{E}]\right),
\end{aligned}
$$

where $c_{x}=\left(1+\frac{1}{2}\right)=\frac{3}{2}$ is the central charge of the super-Virasoro algebra for the free scalar superfield. Combining (4.37), (4.38), and (4.40) gives

$$
\begin{aligned}
Z_{\chi}=\sum_{s} \int & \operatorname{D} \Phi[d T]\left(\frac{\operatorname{sdet}^{\prime}\left(\hat{D}_{\alpha} \hat{D}^{\alpha}\right)_{\hat{E}}}{\int \hat{E} d^{2} z}\right]^{-D / 2} \\
& \times \frac{\left[\left(\operatorname{sdet} \hat{P}^{\dagger} \hat{P}\right)_{\hat{E}}\right]^{1 / 2}}{\Omega_{\hat{E}}(\{\operatorname{SCK}\})} \\
& \times \exp \left[\frac{D-10}{32 \pi} S_{\mathrm{SL}}[\Phi, \hat{E}]\right],
\end{aligned}
$$

where we have introduced the measure

$$
[d T] \equiv \frac{\operatorname{sdet}\left\langle\Psi^{(i)} \mid \frac{\partial \hat{E}_{\beta}^{M}}{\partial T_{j}} \widehat{E}_{M}^{a}\right\rangle_{\hat{E}}}{\left|G_{s}\right|\left(\operatorname{sdet}\left\langle\Psi^{(i)} \mid \Psi^{(j)}\right\rangle_{\hat{E}}\right)^{1 / 2}} \prod_{i} d T_{i}
$$

Again, the appearance of the superconformal superfield $\Phi$ is restricted to $S_{\mathrm{SL}}[\Phi]$.

As for the case of bosonic world sheets, it is possible to define a critical exponent for the sum over random supersurfaces. Since the area of the world sheet, $\int \sqrt{g} d^{2} \xi$, is not invariant under local supersymmetry we must identify some other parameter to serve as an invariant under superdiffeomorphisms. The natural counting of the number of random supersurfaces is given by

$$
\begin{aligned}
& Z_{\chi}=\int_{0}^{\infty} d L F_{\chi}(L) \\
& F_{\chi}(L)=\sum_{s} \int \frac{\mathrm{D} E \mathrm{D} X}{\mathbf{\Omega}_{E}(\{\mathrm{~L}\} \otimes\{\mathrm{SD}\})} \exp (-S[E, X]) \\
& \quad \times \delta\left[\int E d^{2} z-L\right),
\end{aligned}
$$

where $L$ has the dimension of length instead of area. For fixed spin structure and fixed super-Teichmueller parameters, we evaluate the functional $\Phi$ integral in the saddlepoint approximation. The classical minimum is given by

$\delta S_{\mathrm{SL}}=\int d^{2} z \hat{E} e^{-\Phi} Y\left(e^{\Phi / 2} E_{\alpha}^{M}\right) \delta \Phi=0$

under the constraint

$$
\int d^{2} z \hat{E} e^{-\Phi} \delta \Phi=0 \text {. }
$$

This is satisfied if, and only if, $Y\left(e^{\Phi / 2} E_{\alpha}^{M}\right)$ is a constant superfield. In terms of component fields, this implies that only the bosonic component $A$ of the constant superfield $Y$ is nonzero. As defined in Appendix B, $A$ is the auxiliary field of the supervierbein. Explicitly, in the WessZumino gauge

$$
E_{m}^{a}=e_{m}^{a}+i \bar{\theta} \gamma^{a} \psi_{m}+\frac{1}{4}\left(i \bar{\theta} \theta e_{m}^{a} A\right),
$$

where $e_{m}^{a}$ is the zweibein, and $\psi_{m}$ is the gravitino field. For the bosonic case, it is well known that for any fiducial reference metric $\hat{g}$ on a two-dimensional manifold, there exists a conformal field $\phi$ such that $R\left(e^{-\phi} \hat{g}\right)=$ const, and $\phi$ is unique up to an additive constant. Here we shall simply propose the following conjecture: for any supervierbein $E_{A}^{M}$ satisfying the kinematic constraints, there exists a superconformal transformation $E_{A}^{M} \rightarrow E_{A}^{M^{\prime}}$, such that

$$
Y\left(E_{A}^{M^{\prime}}\right)=\text { const . }
$$

This is easily established at the linearized level, as can be seen by reverting to component fields. Assuming this statement, the rest of the calculation parallels that given in the bosonic case:

$$
\left.F_{\chi}(L)=L^{[(D-10) / 8] \chi} \int[d T] \Delta(T) e^{-B(T)} e^{-S_{\mathrm{SL}}\left(\Phi_{1}\right)}\left\{\operatorname{sdet}^{\prime}\left[\left(D_{\alpha} D^{\alpha}+\frac{4 \pi \chi}{L}\right]\right] E_{L}\right\}^{-1 / 2} \iint^{2} E_{L} d^{2}(z)\right]^{-1 / 2}
$$

where $E_{L}=\widehat{E} e^{-\Phi_{L}}$ and $\Phi_{L}$ is the classical solution given by (4.44) and (4.45) satisfying

$$
\int \hat{E} e^{-\Phi_{L}} d^{2} z=L
$$

Then, 


$$
\begin{aligned}
& {\left[\operatorname{sdet}^{\prime}\left(D_{\alpha} D^{\alpha}+\frac{4 \pi \chi}{L}\right]_{E_{L}}\right]^{-1 / 2}\left[\int E_{L} d^{2} z\right]^{1 / 2}=\left[\operatorname{set}^{\prime}\left(D_{\alpha} D^{\alpha}\right)_{E_{L}}\right]^{-1 / 2}\left[\int E_{L} d^{2} z\right]^{1 / 2}} \\
& \times \exp \left[-\frac{1}{2} \operatorname{str} \ln \left[1+\left(D_{\alpha} D^{\alpha}\right)_{E_{L}}^{-1} \frac{4 \pi \chi}{L}\right]\right] \\
& \underset{L \rightarrow \infty}{\sim} L^{(3 / 2) \chi / 12}\left(\operatorname{sdet}^{\prime} D_{\alpha} D^{\alpha}\right) \quad\left[\int E_{1} d^{2} z\right]^{1 / 2} \exp \left[B^{\prime}(T)\right]
\end{aligned}
$$

In contrast to the bosonic case, the exponent in the second factor does not give a $\ln (L)$ dependence, because of supersymmetry. Notice that the asymptotic power behavior

$$
F_{\chi}(L) \underset{L \rightarrow \infty}{\sim} L^{[(D-9) / 8] \chi-1}
$$

is completely independent of the spin structure, which only affects the coefficient $C_{\chi}$ in $F_{\chi}(L)$. It is easy to generalize this argument to the case of arbitrary superconformal fields on the super world sheet. We have

$$
b_{\chi}=\left(\frac{\sum c_{i}}{12}-\frac{9}{8}\right) \chi-1,
$$

where the $c_{i}$ 's are the central charges of the superVirasoro algebra for the superconformal fields introduced on the world sheet.

We emphasize that thus far our discussion dealt with properties of the fermionic string path integral that utilize only the local features of moduli space, which makes possible a treatment outside of the critical dimension. However, as is shown in Sec. VI, to obtain the Gliozzi-ScherkOlive spacetime supersymmetric projection necessitates an independent summation over spin structures separately for the left-handed and right-handed spinors. It is only in the critical dimension that the required factorization of the fermion determinant into the product of its lefthanded and right-handed sectors can be done without the introduction of a global anomaly.

\section{THE HETEROTIC STRING IN A CHIRAL SUPERSPACE}

Heterotic strings share several properties of the bosonic and NSR string models with an important exception: the heterotic string has chiral fields and as a consequence, gravitational, or local Lorentz anomalies can arise on the world sheet. Demanding the absence of the gravitational anomaly forces the model to the critical dimension, $D=10$, as is demonstrated in Ref. 19 . Therefore, the discussion in this section is confined to the case of the heterotic string in its critical dimension. The path-integral formulation for the heterotic string in the
Wess-Zumino gauge has been discussed by Moore, Nelson, and Polchinski. ${ }^{9}$ Here, we examine the problem of constructing the path integral in superspace with manifest invariance under superdiffeomorphisms.

The definition of the measure in functional space in general breaks superconformal as well as Lorentz invariance giving rise to both superconformal and Lorentz anomalies. In ten dimensions and with 32 left-handed chiral fermion fields these anomalies are absent. However, there remains the more serious difficulty of defining chiral determinants in supermoduli space. In the absence of a proof of holomorphic factorization, there is the possibility of a global obstruction to factorization which renders the fermionic determinant ill defined. Here we restrict ourselves to a discussion of the measure, stopping short of a complete expression for the path integral. Since the formulation here closely parallels Secs. II and IV we will be brief in our discussion.

The chiral nature of the model makes it useful to introduce the complex tensor calculus as described in Appen$\operatorname{dix}$ B. In complex tensor notation, ${ }^{2,3}$ the helicity +1 and -1 superfields are defined by

$$
\begin{aligned}
& \Phi_{z}=\Phi^{\bar{z}}=\left(\Phi_{1}-i \Phi_{2}\right) / \sqrt{2}, \\
& \Phi_{\bar{z}}=\Phi^{z}=\left(\Phi_{1}+i \Phi_{2}\right) / \sqrt{2},
\end{aligned}
$$

where $z=\xi^{1}+i \xi^{2}$, and the right-handed fermionic coordinate $\theta$ carries $+\frac{1}{2}$ helicity. Then,

$$
\begin{aligned}
& \delta^{z \bar{z}}=\delta^{\bar{z} z}=1, \quad \delta^{z z}=\delta^{\bar{z} z}=0, \\
& \gamma_{++}^{z}=-\sqrt{2}, \quad \gamma_{--}^{\bar{z}}=\sqrt{2} .
\end{aligned}
$$

In complex notation, the partition function for a given topology of the base manifold $M$ is given by ${ }^{9}$

$$
\begin{aligned}
& Z_{\chi}=\sum_{s} \int_{\mathrm{m}_{H}} \mathrm{D} E_{M}^{A} \mathbf{D} X^{I} \mathbf{D} \Lambda_{J} \exp \left(-S_{H}[E, X, \Lambda]\right) \\
& S_{H}=\int_{M_{H}}\left(i \sqrt{2} \nabla_{\bar{z}} X^{I} \nabla_{+} X^{I}+\lambda_{J} \nabla_{+} \lambda_{J}\right) E d^{2} \xi d \theta
\end{aligned}
$$

where $E_{M}^{A}, X^{I}$, and $\Lambda_{J}$ are $N=\frac{1}{2}$ superfields defined on the heterotic manifold $M_{H}$. The integration is over the space of heterotic supervielbeins, $\mathbf{m}_{H} . E$ is the super- 
determinant of the supervierbein $E_{M}^{A}$. The heterotic manifold, $M_{H}$, is a right-handed chiral spin bundle with spin structure $s$ constructed on the base manifold $M$. $Z=\left(\xi_{1}, \xi_{2} ; \theta\right)=(z, \bar{z} ; \theta)$, with $\xi_{m}$ and $\theta$ the coordinates of the base manifold and the fiber, respectively. The fields $\Lambda_{J}(J=1,2, \ldots, 32)$ are left-handed Majorana-Weyl fermions as defined in Appendix A. The covariant derivative is given by

$$
\nabla_{A}=E_{A}^{M} \partial_{M}+\Omega_{A} L
$$

where $\Omega_{A}$ is the heterotic spin connection, and the curvature is related to the torsion as follows:

$$
\left[\nabla_{A}, \nabla_{B}\right\}=-T_{A B}^{C} \nabla_{C}+R_{A B} L .
$$

The number of independent components of the supervierbein are limited by the kinematic constraints imposed on the torsion:

$$
\begin{aligned}
& T_{++}^{z}=2 i \gamma_{++}^{z}, \\
& T_{++}^{\bar{z}}=T_{++}^{+}=T_{\bar{z}+}^{A}=T_{+_{+}}^{+}=0 .
\end{aligned}
$$

Solving these constraints and the Bianchi identities, all components of the heterotic torsion and heterotic curvature can be expressed in terms of a single superfield $R_{\bar{z}+}$, analogous to the superfield $Y$, in the case of the NSR string. The seven kinematic constraints determine the three heterotic spin connections in terms of the supervielbein and provide four relations among the $E_{A}^{M}$.

Since only five of the nine heterotic vielbein fields are independent, we take the following five fields to span the displacements $\delta E_{A}^{M}$ :

$$
\delta H_{\bar{z}}^{\bar{z}}, \delta H_{+}^{+}, \delta H_{+}^{z}, \delta H_{\bar{z}}^{z}, \text { and } \delta H_{+}^{\bar{z}},
$$

where $\delta H_{A}^{B} \equiv\left(\delta E_{A}^{M}\right) E_{M}^{B}$. The action $S_{H}$ in (5.3), is invariant under local Lorentz transformations because the volume element $E d^{2} \xi d \theta$ carries a lower, right-handed spinor index $d \theta$ while the integrand carries its complement. The measure for $\mathbf{D} E_{M}^{A}$ may be defined by a Lorentz-invariant metric:

$$
\begin{aligned}
\left\|\delta E_{A}^{M}\right\|^{2}=\int[ & \left(c_{1} \delta H_{\bar{z}}^{\bar{z}}+c_{2} \delta H_{+}^{+}\right) \delta H_{+}^{z} \\
& \left.+c_{3} \delta H_{\bar{z}}^{z} \delta H_{+}^{\bar{z}}\right] E d^{2} \xi d \theta,
\end{aligned}
$$

where $c_{1}, c_{2}$, and $c_{3}$ are arbitrary coefficients. This metric is however degenerate. There does not exist a nondegenerate, local Lorentz-invariant metric for $\delta H$. Similarly, the measures for $\mathbf{D} X^{I}$ and $\mathbf{D} \Lambda_{J}$ cannot be defined by a Lorentz-invariant metric.

We choose to abandon manifest Lorentz invariance while maintaining manifest invariance under diffeomorphisms and local supersymmetry transformations. In doing so, we make the assumption that the Lorentz anomaly does not reappear. A rigorous usage of this formalism would necessitate explicitly checking the final expressions for Lorentz invariance. Thus, we define the measure for $\mathbf{D} E_{M}^{A}$ as follows:

$$
\begin{aligned}
\left\|\delta E_{A}^{M}\right\|^{2}=\int & {\left[\left(\delta H_{\bar{z}}^{\bar{z}}\right)^{2}+c_{1}\left(\delta H_{+}^{+}\right)^{2}\right.} \\
& \left.+c_{2}\left(\delta H_{+}^{z}\right)^{2}+c_{3} \delta H_{\bar{z}}^{z} \delta H_{+}^{z}\right] E d^{2} \xi d \theta,
\end{aligned}
$$

where $c_{1}, c_{2}$, and $c_{3}$ are arbitrary coefficients. In order to perform an orthogonal decomposition of the space of infinitesimal variations $\delta E_{A}^{M}$, it is essential that a positivedefinite metric can be defined on this space as was done for the superstring. Again, a suitable choice of gauge slice may resolve this problem.

As an aside, it is interesting to compare the definitions of the measure for the different string path integrals. To put them on an equal footing introduce zweibeins $e_{m}^{a}$ for the bosonic string as well. In complex tensor notation, the four independent fields that span the variations $\delta e_{m}^{a}$ are $H_{z}^{z}, H_{\bar{z}}^{\bar{z}}, H_{\bar{z}}^{z}$, and $H_{z}^{\bar{z}}$. The first two can be gauged away by conformal and Lorentz transformations, and the last two by diffeomorphisms. The heterotic string has nine $N=\frac{1}{2}$ supervielbeins, four of which are supersymmetric generalizations of the zweibeins, and the additional five are $H_{+}^{z}, H_{+}^{\bar{z}}, H_{z}^{+}, H_{\bar{z}}^{+}$, and $H_{+}^{+}$. Of these nine fields, only five are independent. The independent set in (5.7) is chosen such that the measure involves a minimal number of differential operators. For the superstring, there are sixteen $N=1$ supervierbeins, nine of which are supersymmetric generalizations of the heterotic supervielbeins, and the additional seven are $H_{-}^{z}, H_{-}^{\bar{z}}$, $H_{z}^{-}, H_{\bar{z}}^{-}, H_{-}^{+}, H_{+}^{-}$, and $H_{-}^{-}$. Of these sixteen fields, only six are independent. The independent set in (4.6) is chosen so that the operators involved in the measure of the functional integral have the lowest possible dimension.

The rest of the discussion closely parallels that given for the superstring. The heterotic diffeomorphism group \{HD\} is defined as the set of differentiable mappings from $M_{H}$ onto itself:

$$
\begin{aligned}
& Z \in M_{H} \rightarrow Z^{\prime} \in M_{H}, \\
& E_{M}^{A}(Z) \rightarrow E_{M}^{A^{\prime}}(Z), \quad E_{M}^{A^{\prime}}\left(Z^{\prime}\right) d Z^{M^{\prime}}=E_{M}^{A}(Z) d Z^{M}, \\
& X^{I}(Z) \rightarrow X^{I^{\prime}}(Z), \quad X^{I^{\prime}}\left(Z^{\prime}\right)=X^{I}(Z), \\
& \Lambda_{J}(Z) \rightarrow \Lambda_{J}^{\prime}(Z), \quad \Lambda_{J}^{\prime}\left(Z^{\prime}\right)=\Lambda_{J}(Z) .
\end{aligned}
$$

The local Lorentz group $\{L\}$, is the set of local Lorentz transformations:

$$
\begin{aligned}
& X^{I}(Z) \rightarrow X^{I}(Z) \\
& E_{M}^{\bar{z}}(Z) \rightarrow \exp [i \Theta(Z)] E_{M}^{\bar{z}}(Z), \\
& E_{M}^{+}(Z) \rightarrow \exp \left\{\frac{1}{2}[i \Theta(Z)]\right\} E_{M}^{+}(Z), \\
& \Lambda_{J}(Z) \rightarrow \exp \left\{\frac{1}{2}[i \Theta(Z)]\right\} \Lambda_{J}(Z) .
\end{aligned}
$$

The group $\{\mathrm{HC}\}$ is the set of (heterotic) conformal transformations:

$$
\begin{aligned}
& E_{M}^{z} \rightarrow \Lambda E_{M}^{z}, \\
& E_{M}^{\bar{z}} \rightarrow \Lambda E_{M}^{\bar{z}}, \\
& E_{M}^{+} \rightarrow \Lambda^{1 / 2} E_{M}^{+}-\left[i E_{M}^{z}\left(\nabla_{+} \Lambda\right)\right] / \sqrt{2} .
\end{aligned}
$$

Factoring out the subgroup $\left\{\mathrm{HD}_{0}\right\}$ from $\{\mathrm{HD}\}$, where $\left\{\mathrm{HD}_{0}\right\}$ is the set of heterotic diffeomorphisms that may be expressed as successive operations of infinitesimal transformations, we can define the factor group 
$G_{H}=\{\mathrm{HD}\} /\left\{\mathrm{HD}_{0}\right\}$. However, $G_{H}$ is simply $\cong\{D\} /\left\{D_{0}\right\}$, since the supermanifold has no topology in the odd directions.

Let $\mathbf{m}_{H}$ be the space of all heterotic supervielbeins compatible with the kinematic constraints. We consider a gauge slice $S_{H}^{\prime}$ that represents the coset space $\mathbf{m}_{H}^{\prime} \equiv \mathbf{m}_{H} /\{\mathbf{L}\} \otimes\left\{\mathbf{H D}_{0}\right\}$. Introducing a representation $S_{H}=\left\{\widehat{E}_{M}^{A}(Z ; T)\right\}$ for $\mathbf{m}_{H}^{\prime} /\{\mathrm{HC}\}$, any element of $S_{H}^{\prime}$ can be written as

$$
\begin{aligned}
E_{M}^{z} & =\exp [\Phi(Z)] \widehat{E}_{M}^{z}(T ; Z) \\
E_{M}^{\bar{z}} & =\exp [\Phi(Z)] \widehat{E}_{M}^{\bar{z}}(T ; Z) \\
E_{M}^{+}= & \exp \left[\frac{1}{2} \Phi(Z)\right] \widehat{E}_{M}^{+}(T ; Z) \\
& -\left\{i \exp [\Phi(Z)] \nabla_{+} \Phi \hat{E}_{M}^{z}(T ; Z)\right\} / \sqrt{2}
\end{aligned}
$$

Variations $\delta \widehat{E}_{A}^{M}$ around an element, $\widehat{E}_{A}^{M} \in S_{H}$, can be written in the following form:

$$
\left(\begin{array}{c}
\delta H_{\bar{z}}^{\bar{z}} \\
\delta H_{+}^{+} \\
\delta H_{+}^{z} \\
\delta H_{\bar{z}}^{z} \\
\delta H_{+}^{\bar{z}} \\
\delta C_{i}
\end{array}\right)=\left(\begin{array}{cccccc}
1 & * & * & * & * & * \\
0 & 1 & * & * & * & * \\
0 & 0 & -2 \sqrt{2} i & * & * & * \\
0 & 0 & 0 & -\nabla_{\bar{z}} & * & * \\
0 & 0 & 0 & 0 & -\nabla_{+} & * \\
0 & 0 & 0 & 0 & 0 & J^{i j}
\end{array}\right)\left(\begin{array}{c}
\delta \Phi+i \delta K \\
(\delta \Phi-i \delta K) / 2 \\
\delta Q^{+} \\
\delta Q^{z} \\
\delta Q^{\bar{z}} \\
\delta T_{i}
\end{array}\right) .
$$

Here

$$
\left\langle\Psi^{(i)} \mid \Psi^{(l)}\right\rangle_{E} J_{l}^{j}=\left\langle\Psi^{(i)} \mid \frac{\partial \widehat{E}_{\beta}^{M}}{\partial T_{j}} \widehat{E}_{M}^{a}\right\rangle_{E} .
$$

$\delta Q^{A}$ and $\delta K$ are the variations due to heterotic diffeomorphisms and Lorentz transformations, respectively. Since our choice of the metric for the subspace spanned by $\delta H_{\bar{z}}^{z}$ and $\delta H_{+}^{z}$ in (5.7) is Lorentz invariant, $J_{k}^{j}$ does not suffer from the difficulties of a non-Lorentzinvariant measure. Explicitly, $\left\{\Psi^{(i)}\right\}$ is a basis for $(\operatorname{Im} P)^{\perp}$, where the operator $P$ is given by

$$
P: V^{z} \oplus V^{\bar{z}} \rightarrow V_{\bar{z}}^{z} \oplus V_{+}^{\bar{z}},
$$

and $V^{z}, V^{\bar{z}}, V_{\bar{z}}^{z}$, and $V_{+}^{\bar{z}}$ belong to functional spaces of infinitesimal tensors with helicity $1,-1,2$, and $-\frac{3}{2}$, respectively. Thus,

$$
P \equiv \nabla_{\bar{z}} \oplus \nabla_{+} \quad .
$$

The space $(\operatorname{Im} P)^{\perp}$ is given by

$$
(\operatorname{Im} P)^{\perp}=\left\{\delta \eta_{\bar{z}}^{z} ; \nabla_{+} \delta \eta_{\bar{z}}^{z}=0\right\} \oplus\left\{\delta \eta_{+}^{\bar{z}} ; \nabla_{\bar{z}} \delta \eta_{+}^{\bar{z}}=0\right\},
$$

and $\operatorname{ker} P$ is given by

$$
\begin{aligned}
& \left(\nabla_{\bar{z}} \delta Q\right)_{\bar{z}}^{z}=\nabla_{\bar{z}} \delta Q^{z}=0, \\
& \left(\nabla_{+} \delta Q\right)_{+}^{\bar{z}}=\nabla_{+} \delta Q^{\bar{z}}=0 .
\end{aligned}
$$

To illustrate the meaning of the above formalism, consider the case of the torus (a parallelogram with opposite sides identified in the complex plane). By introducing component fields for $\delta Q^{z}$ and $\delta Q^{\bar{z}},(5.16)$ can be rewritten as

$$
\nabla_{\bar{z}} \Phi^{z} \equiv \frac{\partial}{\partial \bar{z}}\left(\Phi^{z}+\theta \psi_{+}^{z}\right)=0
$$

or

$$
\frac{\partial}{\partial \bar{z}} \Phi^{z}=\frac{\partial}{\partial \bar{z}} \psi_{+}^{z}=0
$$

Likewise, (5.17) gives

$$
\left(\frac{\partial}{\partial \theta}+\theta \frac{\partial}{\partial z}\right)\left(\Phi^{\bar{z}}+\theta \psi_{+}^{\bar{z}}\right)=0
$$

or

$$
\frac{\partial}{\partial z} \Phi^{\bar{z}}=\psi_{+}^{\bar{z}}=0
$$

The solution to Eq. (5.19) is $\Phi^{z}=\Phi^{z}(z)$. Since the only doubly periodic analytic functions on the complex plane are constants, (5.19) and (5.20) imply that $\Phi^{z}$ and $\Phi^{\bar{z}}$ are constant superfields. They are to be interpreted as conformal Killing vectors. Similarly, the only conformal Killing spinor solution to (5.19) is a constant spinor $\psi_{+}^{z}$. For the doubly periodic boundary condition, $\operatorname{sdim}(\operatorname{ker} P)=(2,1)$. For other choices of the boundary condition, $\psi_{+}^{z}=0$, so that $\operatorname{sdim}(\operatorname{ker} P)=(2,0)$.

Using similar arguments for (5.15), we have $\operatorname{sdim}\left[(\operatorname{Im} P)^{\perp}\right]=(2,1)$ for the doubly periodic boundary condition, and $\operatorname{sdim}\left[(\operatorname{Im} P)^{\perp}\right]=(2,0)$ for the other boundary conditions. For $h>1$, the Riemann-Roch theorem suggests

$$
\begin{aligned}
& \operatorname{sdim}(\operatorname{ker} P)=(0,0), \\
& \operatorname{sdim}\left[(\operatorname{Im} P)^{\perp}\right]=(6(h-1), 2(h-1)),
\end{aligned}
$$

which can be confirmed by going to the Wess-Zumino gauge.

\section{SPIN STRUCTURE AND MODULAR INVARIANCE ON THE TORUS}

In this section we examine the partition function for surfaces topologically equivalent to the torus $(\chi=0)$ in the 
context of various string models. ${ }^{18}$ The explicit evaluation of the one-loop partition function both clarifies the formal presentation given above, and also demonstrates the powerful constraints imposed by modular invariance on string models.

The partition function and the critical exponent for the bosonic string are immediately obtained from Eq. (2.34). Below the critical dimension

$$
Z=\sum_{\chi} \int_{0}^{\infty} d A F_{\chi}(A)
$$

and the number of random surfaces with $\chi=0$ and of fixed area $A$, behaves like

$$
F_{0}(A) \underset{A \rightarrow \infty}{\sim} C_{0} A^{-1} e^{K A},
$$

where

$$
C_{0}=V \int[d \tau]\left[\frac{\operatorname{det}^{\prime} \Delta_{\hat{g}}}{\int \sqrt{\hat{g}} d^{2} \xi}\right]^{-D / 2}\left(\operatorname{det}^{\prime} \hat{P}^{\dagger} \hat{P}\right)^{1 / 2} .
$$

The integration domain in moduli space is the parallelogram shown in Fig. 3(a). By rescaling the domain to $0 \leq \xi^{m} \leq 1 \quad(m=1,2)$, the flat metric can be chosen as

$$
\hat{g}_{m n}=\frac{1}{\tau_{2}}\left(\begin{array}{cc}
1 & \tau_{1} \\
\tau_{1} & \tau^{2}
\end{array}\right),
$$

where $\tau=\tau_{1}+i \tau_{2}$ are the complex moduli, $\tau^{2}=\tau_{1}^{2}+\tau_{2}^{2}$, and the normalization is fixed so that the metric

$$
\int_{M} d^{2} \xi \sqrt{\hat{g}}=1
$$

is independent of the moduli $\tau$. To calculate the partition function, we choose a basis $\left\{\psi_{m n}^{(i)}\right\}$ which spans $\operatorname{ker} P_{1}^{\dagger}$, where $\hat{g}^{m n} \psi_{m n}^{(i)}=0$. To be specific, let us choose

$$
\psi_{m n}^{(1)}=\left(\begin{array}{cc}
0 & 1 \\
1 & 2 \tau_{1}
\end{array}\right), \quad \psi_{m n}^{(2)}=\left(\begin{array}{cc}
-1 & 0 \\
0 & +\tau^{2}
\end{array}\right),
$$

so that

$$
\begin{aligned}
& \operatorname{det}\left\langle\psi^{(i)} \mid \frac{\partial \hat{g}}{\partial \tau_{j}}\right\rangle=+\frac{4}{\tau_{2}}, \\
& \operatorname{det}\left\langle\psi^{(i)} \mid \psi^{(j)}\right\rangle=4 \tau_{2}^{2},
\end{aligned}
$$

and the volume element given by (2.35),

$$
\int[d \tau]=\int_{F} 2 \frac{d \tau_{1} d \tau_{2}}{\tau_{2}^{2}},
$$

is modular invariant. The determinants in (6.3) can be rewritten as

$$
\begin{aligned}
& \left(\operatorname{det}^{\prime} \hat{P}_{1}^{\dagger} \hat{P}_{1}\right)^{1 / 2}\left(2 \pi \operatorname{det}^{\prime} \Delta_{\hat{g}}\right)^{-D / 2} \\
& \quad=\frac{1}{2}(2 \pi)^{-D / 2}\left(\operatorname{det}^{\prime} \Delta_{\hat{g}}\right)^{(1-D / 2)} .
\end{aligned}
$$

The functional determinant $\operatorname{det}^{\prime} \Delta_{\hat{g}}$ is manifestly modular invariant and has been evaluated: ${ }^{4}$

$$
\begin{aligned}
\operatorname{det}^{\prime}\left(\Delta_{\hat{g}}\right) & =e^{-\pi \tau_{2} / 3} \tau_{2}\left|\prod_{n=1}^{\infty}\left(1-e^{2 \pi i n \tau}\right)\right|^{4} \\
& =A^{*}(\tau)^{2} A(\tau)^{2}
\end{aligned}
$$

with $\mathbf{A}(\tau)$ defined as

$$
\begin{aligned}
A(\tau) & =\left(\frac{-i \tau}{|\tau|}\right)^{1 / 4} e^{\pi i \tau / 12} \tau_{2}^{1 / 4} \prod_{n=1}^{\infty}\left(1-e^{2 \pi i \tau n}\right) \\
& =\left(\frac{-i \tau}{|\tau|}\right)^{1 / 4} \tau_{2}^{1 / 4} \eta(\tau)
\end{aligned}
$$

Here, $\eta(\tau)$ is the Dedekind eta function. The phase of $A(\tau)$ is chosen such that $A(-1 / \tau)=A(\tau)$. It is important to point out that the exponential factor $e^{-\pi \tau_{2} / 3}$ in (6.11), which is related to the value of the Regge intercept, and the presence of the tachyonic mode, is uniquely fixed by modular invariance. The partition function in the critical dimension $(D=26)$ is

$$
Z_{(\chi=0)}=\int_{F} \frac{d \tau_{1} d \tau_{2}}{2 \tau_{2}^{2}}(2 \pi)^{-13}|A(\tau)|^{-48},
$$

where $F$ is the fundamental domain $\left[-\frac{1}{2}<\tau_{1}\right.$ $\left.\leq \frac{1}{2} ; \tau_{2}>0, \quad|\tau|>1\right]$. The extra factor of $\left(\frac{1}{2}\right)$ comes from the discrete symmetry $\xi_{m} \rightarrow-\xi_{m}$. For $D<26$, the critical exponent is

$$
b_{0}=-1,
$$

and the coefficient $C_{0}$ is given by

$$
C_{0}=V \int_{F} \frac{d \tau_{1} d \tau_{2}}{2 \tau_{2}^{2}}(2 \pi)^{-(D-1) / 2}|A(\tau)|^{-2 D} .
$$

Because of the presence of the tachyon, this integral is not well defined: $|A(\tau)|^{-2 D}$ diverges exponentially for large values of $\tau_{2}$. In other words, the partition function is dominated by very thin "tubes," and this system cannot describe a system of random surfaces.

Next, we introduce $N$, massless, free fermion fields $\lambda_{J}^{\alpha}$ $(\alpha=1,2)$ on the world sheet. Consider the case of $N$ lefthanded fermions belonging to a $\mathrm{O}(N)$ vector multiplet. The action is of the form

$$
S=\int d^{2} \xi \sqrt{g} g^{m n}\left(\partial_{m} X^{I} \partial_{n} X^{I}+\sum_{J=1}^{N} \bar{\Lambda}_{J} \gamma^{m} \partial_{m} \Lambda_{J}\right),
$$

and the partition function is given by

$$
\begin{aligned}
Z=\sum_{s} \int & {\left[\prod_{N} \mathbf{D} \lambda_{J}^{\alpha}\right]\left[\mathbf{D} X^{I}\right]\left[\mathbf{D} e_{m}^{a}\right] } \\
& \times \exp (-S[\lambda, X, e])
\end{aligned}
$$

The result of the fermionic integration, including the sum over spin structures, is the factor 


$$
\begin{aligned}
K_{L}= & \left(\operatorname{det} D_{L}\right)_{P P}^{N}+\left(\operatorname{det} D_{L}\right)_{A P}^{N}+\left(\operatorname{det} D_{L}\right)_{P A}^{N} \\
& +\left(\operatorname{det} D_{L}\right)_{A A}^{N},
\end{aligned}
$$

where $P$ denotes periodic and $A$ the antiperiodic boundary condition on the spinor fields in the integration region shown in Fig. 3. Of course, the above expression is multiplied by the determinants coming from the integration of $e_{m}^{a}$ and $X^{I}$; since these are already modular invariant, we need only check the modular invariance of the fermionic determinants. For the $(P P)$ case $\lambda_{I}$ has a zero mode $\lambda_{I 0}$. Integration over each of these zero modes gives zero so that

$$
\left(\operatorname{det} D_{L}\right)_{P P}^{N}=0 .
$$

For the next term, $\left(\operatorname{det} D_{L}\right)_{A P}^{N} \cong\left(\operatorname{det} \Delta_{\hat{g}}\right)_{A P}^{N / 2}$. This determinant is easy to evaluate. First we enlarge the integration domain from $(\tau, 1)$ to $(2 \tau, 1)$ as illustrated in Fig. 3(b). The corresponding determinant includes modes that are both periodic and antiperiodic in the domain $(\tau, 1)$. The periodic modes can be removed by taking the following ratio:

$$
B_{1}^{N} \equiv\left(\operatorname{det} D_{L}\right)_{A P}^{N}=\left(\frac{A(2 \tau)}{A(\tau)}\right)^{N} .
$$

Similar arguments [see Fig. 3(c)] give

$$
B_{2}^{N} \equiv\left(\operatorname{det} D_{L}\right)_{P A}^{N}=\left(\frac{A(\tau / 2)}{A(\tau)}\right)^{N},
$$

where the domain is rescaled so that the coordinate $\xi_{1}$ belongs to the interval $[0,1]$. From the $(P A)$ domain, we can shift $\tau \rightarrow \tau+1$ to obtain the ( $A A)$ domain [see Fig. 3(d)] so that

$$
B_{3}^{N} \equiv\left(\operatorname{det} D_{L}\right)^{N}=\left(\frac{A\left(\tau / 2+\frac{1}{2}\right)}{A(\tau+1)}\right)^{N} .
$$

Since the functional integral determines this function only up to a phase, we have

$$
\begin{aligned}
K_{N}= & B_{1}^{N}+\beta B_{2}^{N}+\alpha B_{3}^{N} \\
= & {\left[\frac{A(2 \tau)}{A(\tau)}\right]^{N}+\beta\left[\frac{A(\tau / 2)}{A(\tau)}\right]^{N} } \\
& +\alpha\left(\frac{A\left(\tau / 2+\frac{1}{2}\right)}{A(\tau+1)}\right)^{N} .
\end{aligned}
$$

Here $\beta$ and $\alpha$ are some phases to be fixed by modular invariance. $K_{N}(\tau)$ should be invariant under modular transformations $\tau \rightarrow(a \tau+b) /(c \tau+d)$, where $a, b, c, d$ are integers and $a d-b c=1$. Using (6.12) for $A(\tau)$, the $B_{i}(\tau)$ transform under modular transformations as follows:

$$
\begin{aligned}
\tau \rightarrow-\frac{1}{\tau}: \quad & B_{1} \rightarrow B_{2}, \\
B_{2} & \rightarrow B_{1}, \\
B_{3} & \rightarrow B_{3},
\end{aligned}
$$

so that $\beta=1$. Likewise,

$$
\begin{aligned}
\tau \rightarrow \tau+1: \quad & B_{1} \rightarrow \exp (i \pi / 12) B_{1}, \\
& B_{2} \rightarrow B_{3}, \\
& B_{3} \rightarrow \exp (-i \pi / 12) B_{2},
\end{aligned}
$$

so that

$$
K_{N} \rightarrow e^{i \pi N / 12}\left(B_{1}^{N}+e^{-i \pi N / 6} \alpha B_{2}^{N}+e^{-i \pi N / 12} B_{3}^{N}\right) .
$$

Therefore, modular invariance requires

$$
\begin{aligned}
& \alpha=e^{+i \pi N / 6}, \\
& N=8 l,
\end{aligned}
$$

where the overall phase in (6.25) may cancel those arising from other fields. If we now consider only right-handed fields, then

$$
K_{N} \rightarrow e^{-i \pi N / 12}\left(B_{1}^{* N}+B_{2}^{* N}+e^{+i \pi N / 4} \alpha^{*} B_{3}^{* N}\right) .
$$

Here $B^{*}$ and $\alpha^{*}$ are the complex conjugates of the corresponding variables in (6.22). Next, let us consider

$$
\begin{aligned}
K_{N_{1} N_{2}}(2)= & {\left[B(1)_{1}^{N_{1}}+B(1)_{2}^{N_{1}}+\alpha_{1} B(1)_{3}^{N_{1}}\right] } \\
& \times\left[B^{*}(2)_{1}^{N_{2}}+B^{*}(2)_{2}^{N_{2}}+\alpha^{* 2} B^{*}(2)_{3}^{N_{2}}\right],
\end{aligned}
$$

where either both factors involve only left-moving fields, or one factor involves only left-moving and the other only right-moving fields. Modular invariance requires

$$
\begin{aligned}
& N_{i}=0 \quad(\bmod 8), \\
& N_{L}-N_{R}=0 \quad(\bmod 24),
\end{aligned}
$$

where $N_{L}\left(N_{R}\right)$ is the total number of left- (right-) moving fields. It is obvious that

$$
\begin{aligned}
K_{N_{1} N_{2}}(1)= & B(1)_{1}^{N_{1}} B(2)_{1}^{N_{2}}+B(1)_{2}^{N_{1}} B(2)_{2}^{N_{2}} \\
& +a_{1} a_{2} B(1)_{3}^{N_{1}} B(2)_{3}^{N_{2}},
\end{aligned}
$$

is also modular invariant. Now a nontrivial modular invariant function can be obtained by taking the difference of $K_{N_{1} N_{2}}(1)$ and $K_{N_{1} N_{2}}(2)$ :

$$
K_{N_{1} N_{2}}^{\prime}=K_{N_{1} N_{2}}(2)-K_{N_{1} N_{2}}(1)
$$

In general, the spin structures can be intricately correlated. This method for the construction of modularinvariant functions with nontrivial coefficients relating different sets of spin structures can be easily extended. Since modular invariance is only a necessary condition for viable string models, other constraints such as demanding a tachyon-free spectrum, a proper space-time projection and the absence of the space-time gravitational anomaly can impose further limitations on the list of interesting models. Properties of string models corresponding to various spin structures will be discussed elsewhere.

Before we apply this analysis to the NSR and the heterotic string models, we observe that for left-rightsymmetric models, the function $K$ is modular invariant for any $N$. Also, for $N=8$, 


$$
K_{8}=B_{1}^{8}+B_{2}^{8}+e^{4 i \pi / 3} B_{3}^{8}=0
$$

which follows from the Jacobi identity.

For the closed superstring in $D$ dimensions,

$$
Z=\sum_{\chi, s} \int \frac{\mathbf{D} e_{m}^{a} \mathbf{D} \psi_{m}}{\Omega_{e}(\{\mathrm{sym}\})} \mathbf{D} \lambda^{I} \mathbf{D} X^{I} e^{-S[X, \lambda, e, \psi]}
$$

where the summation is over different topologies and over all spin structures permitted by the symmetry properties of the model. $\Omega_{e}(\{\mathrm{sym}\})$ is a formal expression for the volume of the superdiffeomorphism and Lorentz groups. (At the critical dimension, the volume of the superconformal group must also be included.) Expressed in terms of component fields, the action is ${ }^{14}$

$$
S(e, \psi, \lambda, X)=\int d^{2} \xi e\left[\frac{1}{2} g^{m n} \partial_{m} X^{I} \partial_{n} X^{I}+\frac{i}{2} \lambda^{I} \gamma^{m} \partial_{m} \lambda^{I}+\frac{i}{2} \psi_{n} \gamma^{m} \gamma^{n} \lambda^{I}\left[\partial_{m} X^{I}-\frac{i}{4} \psi_{m} \lambda^{I}\right]+\frac{F^{2}}{2}\right]
$$

where $F^{I}$ is the auxiliary matter field. For the torus $(\chi=0)$, the sum is over the four boundary conditions shown in Fig. 3. Integrating the $D$-dimensional fields, the partition function for the torus reduces to a product of fermion determinants. For the left-moving sector, the $(P P)$ boundary condition permits a conformal Killing spinor (a spin- $\frac{1}{2}$ spinor) and a supermodulus (a parameter in the form of a constant spin- $\frac{3}{2}$ spinor), while the other boundary conditions $(A P, P A$, and $A A)$ permit neither conformal Killing spinors nor supermoduli. However, the presence of zero modes in the matter fields $\lambda_{L}^{I}$ in the $P P$ case again renders

$$
\left(\operatorname{det} D_{L}\right)_{P P}^{D}=0
$$

so that for the torus, supermoduli do not appear. Integrating over the fermionic fields gives

$$
\begin{aligned}
K_{(\chi=0)}=\sum_{s}\left(\operatorname{det} \hat{P}_{1 / 2}^{\dagger} \hat{P}_{1 / 2}\right)_{L}^{1 / 2}\left(\operatorname{det} D_{L}\right)^{D} \\
\quad \times \sum_{s}\left(\operatorname{det} \hat{P}_{1 / 2}^{\dagger} \hat{P}_{1 / 2}\right)_{R}^{1 / 2}\left(\operatorname{det} D_{R}\right)^{D} .
\end{aligned}
$$

$P_{1 / 2}$ is the operator that converts the spin- $\frac{1}{2}$ spinor variable that appears in the supersymmetry transformation to a spin- $\frac{3}{2}$ variable $\left(\delta \psi_{m}=P_{1 / 2} \psi=\gamma^{n} \gamma_{m} \nabla_{n} \psi\right)$, and $P_{1 / 2}^{\dagger}$ is its adjoint $\left(P_{1 / 2}^{\dagger} \psi=-2 \nabla_{m} \psi^{m}\right)$. For the torus with superconformally flat metric, we have the relation

$$
\begin{aligned}
K_{L} & \equiv \sum_{s}\left(\operatorname{det} \hat{P}_{1 / 2}^{\dagger} \hat{P}_{1 / 2}\right)_{L}^{1 / 2}\left(\operatorname{det} D_{L}\right)^{D} \\
& =\sum_{s}\left(\operatorname{det} D_{L}\right)^{D-2} .
\end{aligned}
$$

Hence, $(D-2)=8 l$ gives modular invariance. For spacetime supersymmetry, $(l=1)$ or $(D=10)$, it follows from (6.34) that the partition function gives zero cosmological constant in space-time at the one-loop level. Thus, the sum over spin structures is simply the space-time supersymmetry projection. This is the original observation of Gliozzi, Scherk, and Olive. ${ }^{12} D=10$ is also the critical dimension. It is meaningless to consider $l>1$, since for $l>1$, the Liouville action will have the wrong sign and the path integral becomes ill defined.

Let us now consider the right-moving fields. If $K_{L}=0$, it may appear that any combination of rightmoving fields is allowed. However, in general we also demand modular invariance for one-loop scattering amplitudes. Hence, the right-hand sector should be invariant under modular transformations except for an overall phase which is required to cancel the phase that arises in the left-hand sector. For type-II superstrings, both the left- and the right-hand side have $l=1$, so that $K_{(\chi=0)}$ in Eq. (6.38) is modular invariant. Observe that $P P$ and $A P$ come from the Ramond sector while $P A$ and $A A$ come from the Neveu-Schwarz sector. The path integral naturally requires a sum over different boundary conditions; this provides precisely the space-time projection that gives the superstring. ${ }^{12}$ Let us fix the right-moving sector to be world-sheet supersymmetric with $l=1$. Examining (6.28), the solutions with left-moving fermion fields are $\mathrm{SO}\left(8 l_{1}\right) \otimes \mathrm{SO}\left(8 l_{2}\right) \otimes \cdots$ such that

$$
\sum l_{i}=1 \quad(\bmod 3) .
$$

By this, we mean theories with blocks of fermions containing, respectively, $8 l_{1}, 8 l_{2}, \ldots$ fields all with their spin structures pinned together but independent of the other blocks. If the fermions contain an $\mathrm{SO}(8)$ multiplet, $K_{L}=0$ from the Jacobi identity. For $n>32$, the central charge becomes positive, so that the Liouville action carries the wrong sign and the path integral is ill defined. The simplest cases that do not contain a $\mathrm{SO}(8)$ multiplet of fermions, are the cases $l=4$ [the multiplet $\mathrm{SO}(32)$ ] and $l_{1}=2, l_{2}=2$ [multiplets $\mathrm{SO}(16) \otimes \mathrm{SO}(16)$ ]. It turns out that they have the same modular function:

$$
\begin{aligned}
K_{[16 \otimes 16]} & =\left[\left(\frac{A(2 \tau)}{A(\tau)}\right]^{16}+\left[\frac{A(\tau / 2)}{A(\tau)}\right]^{16}+e^{2 i \pi / 3}\left[\frac{A(\tau / 2+1 / 2)}{A(\tau+1)}\right]^{16}\right]^{2} \\
& =2\left[\left(\frac{A(2 \tau)}{A(\tau)}\right]^{32}+\left[\frac{A(\tau / 2}{A(\tau)}\right]^{32}+e^{4 i \pi / 3}\left[\frac{A(\tau / 2+1 / 2)}{A(\tau+1)}\right]^{32}\right] \\
& =2 K_{[32]},
\end{aligned}
$$


where the Jacobi identity has been used. As noted in Ref. 13, in the $S O(16) \otimes S O(16)$ case, an $E_{8} \otimes E_{8}$ symmetry is induced. Specifically, the $\mathrm{E}_{8}$ character function is given by ${ }^{20}$

$$
\operatorname{ch}\left(\mathrm{E}_{8 \text { basic }}\right)=\operatorname{ch}(\mathrm{SO}(16))+\operatorname{ch}\left(\mathrm{SO}(16)_{1 / 2}\right),
$$

where $[q \equiv \exp (2 \pi i \tau)]$

$$
\begin{aligned}
& \operatorname{ch}\left(\operatorname{SO}(16)_{1 / 2}\right)=16 q^{1 / 3}\left(\frac{A(2 \tau)}{A(\tau)}\right)^{16}, \\
& \operatorname{ch}(\operatorname{SO}(16))=16 q^{1 / 3}\left[\left(\frac{A(\tau / 2)}{A(\tau)}\right]^{16}+e^{2 i \pi / 3}\left[\frac{A(\tau / 2+1 / 2)}{A(\tau+1)}\right]^{16}\right] .
\end{aligned}
$$

To see this explicitly, we follow the construction of Ref. 21 by exploiting the fact that $\mathrm{SO}(16) \supset \mathrm{SO}(8) \otimes \mathrm{SO}(8)$, where the isometry of the $\mathrm{SO}(8)$ Dynkin diagram permits the $\mathrm{E}_{8}$ construction from $\mathrm{SO}(16)$. Generalizations of these arguments for other gauge groups will be given elsewhere.

After completion of this work, we learned that several papers overlap with our discussion on the spin structure and modular invariance: N. Seiberg and E. Witten [Nucl. Phys. B276, 272 (1986)]; L. Alvarez-Gaume, P. Ginsparg, G. Moore, and C. Vafa [Phys. Lett. 171B, 155 (1986)]; L. Alvarez-Gaume, G. Moore, and C. Vafa [Commun. Math. Phys. 106, 40 (1986)]; L. Dixon and J. Harvey, Princeton report (unpublished); W. Nahm, in Proceedings of the 19th International Symposium: Special Topics in Gauge Field Theories, Ahrenshoop, Germany, 1985 (Institut für Hochenenergiephysik, Akademie der Wissenschaften, Berlin, 1985), p. 303; E. D'Hoker and D.H. Phong [Nucl. Phys. B278, 225 (1986)]. The last paper also formulated the path integral of the NSR string in the component formalism.
Note added. Since the first appearance of this work the question of global obstructions to superholomorphic factorization has been examined by E. D'Hoker and D. Phong [Report No. PUPTHY-1029 (unpublished)]. Using the results of Sec. IV, they have claimed the complete cancellation of anomalies in the critical dimension.

\section{ACKNOWLEDGMENTS}

We are grateful to our colleagues at Cornell for discussions. One of us (S.C.) also thanks B. Ratra for helpful comments. This research was supported by the National Science Foundation.

\section{APPENDIX A}

We summarize the definition of Majorana-Weyl spinors on an (even) $d$-dimensional Euclidean space. Our convention for the metric is

$$
\begin{aligned}
& \left(\gamma_{0}\right)^{2}=-1,\left(\gamma_{1}\right)^{2}=\cdots=\left(\gamma_{d-1}\right)^{2}=+1, \quad\left(\gamma_{5}\right)^{2}=+1 \quad \text { (Minkowski) }, \\
& \left(\gamma_{1}\right)^{2}=\cdots=\left(\gamma_{d}\right)^{2}=+1, \quad\left(\gamma_{5}\right)^{2}=+1 \quad \text { (Euclidean). }
\end{aligned}
$$

Let us begin by considering a Dirac spinor $\psi^{\alpha}$, in a $d$ dimensional space of as yet unspecified metric. The partition function is given by

$$
Z=\int D \bar{\psi} D \psi e^{-S[\bar{\psi}, \psi]},
$$

where

$$
S=\int d^{d} \xi \bar{\psi}\left(\gamma^{m} \partial_{m}+m\right) \psi
$$

There exist two different but equivalent formulations for Dirac spinors. In the first, the fields $\psi$ and $\bar{\psi}$ are taken to be independent, complex, anticommuting variables that satisfy the equations

$$
\begin{aligned}
& \int d \bar{\psi} \bar{\psi}=1, \\
& \int d \psi \psi=1 .
\end{aligned}
$$

The second approach is to make the decomposition
$\psi=\psi_{R}+i \psi_{I}$ where $\psi_{R}$ and $\psi_{I}$ are independent, real, anticommuting variables and the Grassmann integrals are defined as follows:

$$
\begin{aligned}
& \int d \psi_{R} \psi_{R}=1, \\
& \int d \psi_{I} \psi_{I}=1 .
\end{aligned}
$$

In Minkowski (Euclidean) space $\bar{\psi}$ is interpreted as ${ }^{t} \psi^{*} \gamma^{0} \quad\left({ }^{t} \psi^{*} \gamma^{5}\right)$, where $\psi^{*} \equiv \psi_{R}-i \psi_{I}$ is the complex conjugate of $\psi$. Since the operator $i \gamma^{0} \gamma^{m} \partial_{m}\left(i \gamma^{5} \gamma^{m} \partial_{m}\right)$ is Hermitian in Minkowski (Euclidean) space, both formulations are equivalent and one can choose freely between them.

For Weyl fermions, on the other hand, the choice of formulation is not arbitrary. While either formulation works in Minkowski space, only the first can be used in Euclidean space. In Minkowski space the operator $\left\{\gamma^{0} \gamma^{m} \partial_{m}\left[\left(1 \pm \gamma_{5}\right) / 2\right]\right\}$ is still Hermitian, but in Euclidean 
space $\left\{\gamma^{5} \gamma^{m} \partial_{m}\left[\left(1 \pm \gamma_{5}\right) / 2\right]\right\}$ is neither Hermitian nor anti-Hermitian. Thus, $\bar{\psi}$ cannot be regarded as the complex conjugate of $\psi$ if we work in Euclidean space.

We now construct Majorana fermions in Euclidean space using the first formulation. It is well known that, for $d=0,2,4(\bmod 8)$, there exists an antisymmetric matrix $C$ such that the Majorana condition can be satisfied:

$$
\begin{aligned}
& C^{-1} \gamma_{m} C=-\gamma^{m}, \\
& { }^{t} C=-C,
\end{aligned}
$$

and the operator $C\left(\gamma^{m} \partial_{m}+m\right)$ is antisymmetric. Thus, we can construct the Lagrangian

$$
L={ }^{t} \psi C\left(\gamma^{m} \partial_{m}+m\right) \psi \quad, \quad \bar{\psi} \equiv{ }^{t} \psi C
$$

such that

$$
\int \mathbf{D} \psi \exp \left(-\int d^{d} \xi L\right)=\operatorname{Pfaffian}\left(\gamma^{m} \partial_{m}+m\right) .
$$

Here, the $\psi$ 's are complex, anticommuting variables and the measure is defined by

$$
\int d \psi \psi=1 \text {. }
$$

Under a Lorentz transformation, the fields transform as follows:

$$
\psi \rightarrow R \psi, \bar{\psi} \rightarrow \bar{\psi} R^{-1},
$$

where $R$ is the generator of the Lorentz transformation. Then,

$$
{ }^{t}(R) C=C(R)^{-1},
$$

or, equivalently,

$$
{ }^{t} \gamma_{[m}{ }^{t} \gamma_{n]} C=-C \gamma_{[m} \gamma_{n]}
$$

so that the action is Lorentz invariant. Note that this formulation of Majorana fermions is independent of whether the space has a Euclidean or a Minkowski metric.

For $d=2(\bmod 8)$, we define the Lagrangian for Majorana-Weyl fermions by

$$
L \equiv{ }^{t} \psi_{L} C \gamma^{m} \partial_{m} \psi_{L},
$$

with

$$
\left(C \gamma^{m} \partial_{m}\right) \frac{1+\gamma_{5}}{2}=\frac{1+{ }^{t} \gamma_{5}}{2}\left(C \gamma^{m} \partial_{m}\right) .
$$

For example, in two-dimensional Euclidean space the choice

$$
\begin{aligned}
\gamma^{1} & =\left[\begin{array}{ll}
0 & 1 \\
1 & 0
\end{array}\right], \quad \gamma^{2}=\left[\begin{array}{cc}
0 & -i \\
i & 0
\end{array}\right), \\
\gamma^{5} & =\left(\begin{array}{cc}
1 & 0 \\
0 & -1
\end{array}\right), \\
C & =\left(\begin{array}{cc}
0 & -1 \\
+1 & 0
\end{array}\right),
\end{aligned}
$$

satisfies the conditions in (A9).

\section{APPENDIX B}

This appendix contains a useful compendium of facts ${ }^{15}$ related to the discussion in Secs. IV and V. We work on Euclidean world sheets and our convention for the $\gamma$ matrices is given in (A17), with

$$
\left\{\gamma^{m}, \gamma^{n}\right\}=2 \delta^{m n} \text {. }
$$

The bosonic metric is

$$
\delta_{a b}=\operatorname{diag}(1,1), \quad \epsilon_{a b}=-\epsilon_{b a}, \epsilon_{12} \equiv 1 .
$$

Spin indices are raised and lowered by $\epsilon_{\alpha \beta}=\epsilon^{\alpha \beta} ; \epsilon_{12} \equiv-\epsilon_{21}=1$, and

$$
\chi^{\alpha}=\epsilon^{\alpha \beta} \chi_{\beta}, \chi_{\alpha}=-\epsilon_{\alpha \beta} \chi^{\beta} .
$$

The expression $\bar{\psi}_{1} \Gamma \psi_{2} \equiv \psi_{1}^{\alpha} \Gamma_{\alpha}^{\beta} \psi_{2 \beta}$, where $\psi_{1}, \psi_{2}$ are arbitrary spinors, and $\bar{\psi} \equiv{ }^{t} \psi C$. Here $C$ is the matrix defined in (A18). The Fierz rearrangement identity is given by

$$
\left(\bar{\phi}_{1} \phi_{2}\right) \phi_{3}=-\frac{1}{2} \sum_{i}\left(\phi_{1} \Gamma_{i} \phi_{3}\right) \Gamma^{i} \phi_{2}, \quad \Gamma_{i}=\left(1, \gamma_{a}, \gamma_{5}\right) .
$$

A $p$-form is defined by its transformation under the tangent space group:

$$
\delta E^{A}=E^{B} L_{B}^{A} \quad, \quad L_{B}^{A}=L E_{B}^{A},
$$

and

$$
E_{b}^{a}=\epsilon_{b}^{a}, \quad E_{b}^{\alpha}=E_{\beta}^{a}=0, E_{\beta}^{\alpha}=\frac{1}{2}\left(i \gamma_{5}\right)_{\beta}^{\alpha} .
$$

For instance, the superconnection transforms like

$$
\Omega_{\mu B}^{A} \equiv \Omega_{\mu} E_{B}^{A} \quad, \quad \Omega_{m B}^{A} \equiv \Omega_{m} E_{B}^{A},
$$

where $\delta \Omega=-d L$. Tangent-space and world-sheet indices are freely interchanged by the supervierbein $E_{M}^{A}$. The superdeterminant, $E \equiv \operatorname{sdet} E_{M}^{A}$, is defined by

$$
E=\operatorname{det}\left(E_{m}^{a}-E_{m}^{\alpha}\left(E_{\mu}^{\alpha}\right)^{-1} E_{\mu}^{a}\right) / \operatorname{det} E_{\mu}^{\alpha},
$$

and the supertrace is defined as $\operatorname{str} \boldsymbol{M}=\boldsymbol{M}_{a}^{a}-\boldsymbol{M}_{\alpha}^{\alpha}$.

The redundancy in the superfield formulation allows the imposition of the kinematic constraints contained in (4.5). The constraints can be used to demonstrate ${ }^{15}$ that all components of the supercurvature and supertorsion tensors can be expressed in terms of a single scalar superfield, $Y$. The Bianchi identity we make use of is

$$
R_{[A B, C\}}^{D}=D_{[A} T_{B C\}}^{D}+T_{[A B \mid}{ }^{F} T_{F \mid C\}}{ }^{D},
$$

where $T_{[A B\}} \equiv \frac{1}{2}\left[T_{A B}-(-1)^{a b} T_{B A}\right]$ and $(-1)^{A}$ $\equiv \pm 1$, for bosonic and fermionic indices, respectively. The identity $R_{[\alpha \beta \gamma]}^{d}=0$ gives $T_{\beta a}^{b}=0$. Combining the $R_{[\alpha \beta c]}^{d}$ and $R_{[\alpha \beta \gamma]} \delta$ identities gives

$$
\begin{aligned}
& T_{b \gamma}^{\alpha}=\frac{1}{4}\left[\left(i \gamma_{b}\right)_{\gamma}^{\alpha} Y\right], \\
& F_{\alpha \beta}=-i\left(\gamma_{5}\right)_{\alpha \beta} Y,
\end{aligned}
$$

where $Y$ is a scalar superfield. The identities for $R_{[\alpha b c]}{ }^{d}$ and $R_{[\alpha \beta \gamma]}^{\delta}$ yield 


$$
\begin{aligned}
& T_{b c}^{\alpha}=-\frac{1}{4}\left[\epsilon_{b c}\left(i \gamma_{5}\right)^{\alpha \beta} D_{\beta} Y\right], \\
& F_{\alpha b}=-\frac{1}{2}\left[\left(\gamma_{5} \gamma_{b}\right)_{\alpha}^{\beta} D_{\beta} Y\right],
\end{aligned}
$$

and finally the $R_{[a b \gamma\}}^{\delta}$ identity gives

$$
F_{a b}=\frac{1}{4}\left(i \epsilon_{a b} D_{\alpha} D^{\alpha} Y\right)-\frac{1}{4}\left(\epsilon_{a b} Y^{2}\right) .
$$

In the Wess-Zumino gauge the scalar field $Y$ is given by

$$
\begin{array}{r}
Y=A+\bar{\theta} \eta+\frac{\bar{\theta} \theta}{2}\left[R+\frac{1}{2}\left(\bar{\eta} \gamma^{a} \psi_{a}\right)+\frac{1}{4}\left(\epsilon^{a b} i \bar{\psi}_{a} \gamma_{5} \psi_{b} A\right)\right. \\
\left.-\frac{1}{2}\left(A^{2}\right)\right],
\end{array}
$$

where $\psi_{a}$ is the gravitino, and the spinor field $\eta^{\alpha}$ is defined by

$$
\eta^{\alpha}=-2 i \epsilon^{m n} \gamma_{5} D_{m} \psi_{n}^{\alpha}-\frac{1}{2}\left(\gamma^{m} \psi_{m}^{\alpha} A\right)
$$

A similar analysis can be made for heterotic superspace. Here we follow the notation of Ref. 9. A heterotic manifold is constructed as a right-handed chiral spin bundle on the (bosonic) base manifold. It is convenient to work in complex coordinates because of the chiral nature of the heterotic superspace. The bosonic tangent space is parametrized by

$$
z=\xi^{1}+i \xi^{2}, \quad \bar{z}=\xi^{1}-i \xi^{2},
$$

and the metric is defined as

$$
\begin{gathered}
\delta_{z \bar{z}}=\delta_{\overline{z z}}=1, \quad \epsilon_{z \bar{z}}=-\epsilon_{\overline{z z}}=i, \\
\delta_{z z}=\delta_{\overline{z z}}=0, \quad \epsilon_{z z}=\epsilon_{\overline{z z}}=0 .
\end{gathered}
$$

The fermionic tangent space is likewise complexified, with

$$
\epsilon_{+-}=-\epsilon_{-+}=1, \epsilon_{++}=\epsilon_{--}=0 .
$$

Thus $\lambda^{\alpha} \quad(\alpha=1,2) \rightarrow\left(\lambda^{+}, \lambda^{-}\right) \quad$ with $\quad \lambda^{+}=\lambda_{-} \quad$ and $\lambda^{-}=-\lambda_{+}$. The Lorentz generator is defined by

$$
L_{z}^{z}=-L_{\bar{z}}^{\bar{z}}=i, \quad 2 L_{+}^{+}=-2 L_{-}^{-}=i \text {. }
$$

The $\gamma$ matrices in the complex basis are

$$
\gamma^{2}=\left(\begin{array}{cc}
-\sqrt{2} & 0 \\
0 & 0
\end{array}\right), \quad \gamma^{\bar{z}}=\left(\begin{array}{cc}
0 & 0 \\
0 & \sqrt{2}
\end{array}\right), \quad \gamma_{5}=\left(\begin{array}{cc}
i & 0 \\
0 & -i
\end{array}\right) \text {. }
$$

Again, tangent space and space-time indices may be interchanged by the supervierbein

$$
E_{A}^{M}=\left(E_{z}^{M}, E_{\bar{z}}^{M}, E_{+}^{M}\right) .
$$

${ }^{1}$ A.M. Polyakov, Phys. Lett. 103B, 207 (1981).

${ }^{2}$ D. Friedan, in Recent Advances in Field Theory and Statistical Mechanics, proceedings of Les Houches Summer School, Les Houches, France, edited by J.B. Zuber and R. Stora (NorthHolland, Amsterdam, 1984), Vol. 39.

${ }^{3}$ O. Alvarez, Nucl. Phys. B216, 126 (1983).

${ }^{4}$ J. Polchinski, Commun. Math. Phys. 104, 37 (1986).

${ }^{5}$ G. Moore and P. Nelson, Nucl. Phys. B266, 58 (1986).

${ }^{6}$ E. D'Hoker and D.H. Phong, Nucl. Phys. B269, 205 (1986).

${ }^{7}$ A.M. Polyakov, Phys. Lett. 103B, 211 (1981).

${ }^{8}$ E. Martinec, Phys. Rev. D 23, 2604 (1983).

${ }^{9}$ G. Moore, P. Nelson, and J. Polchinski, Phys. Lett. 169B, 47 (1986).

${ }^{10}$ P. DiVecchia, B. Durhuus, P. Olesen, and J. Petersen, Nucl. Phys. B207, 77 (1982); see also D. Friedan, E. Martinec, and S. Shenker, ibid. B271, 93 (1986).

${ }^{11}$ A. Neveu and J.H. Schwarz, Nucl. Phys. B31, 86 (1971); P. Ramond, Phys. Rev. D 3, 2415 (1971).

${ }^{12}$ F. Gliozzi, J. Scherk, and D. Olive, Nucl. Phys. B122, 253 (1977).

${ }^{13}$ D.J. Gross, J.A. Harvey, E. Martinec, and R. Rohm, Phys. Rev. Lett. 54, 502 (1985); Nucl. Phys. B256, 253 (1985); Nucl. Phys. (to be published).

${ }^{14}$ L. Brink, P. DiVecchia, and P. Howe, Phys. Lett. 65B, 471 (1976); S. Deser and B. Zumino, ibid. 65B, 369 (1976).
${ }^{15}$ P. Howe, J. Phys. A 12, 393 (1979).

${ }^{16}$ T. Eguchi and H. Kawai, Phys. Lett. 114B, 247 (1982).

${ }^{17}$ A.B. Zamolodchikov, Phys. Lett. 117B, 87 (1982); A.M. Polyakov, in Differential Geometric Methods in Theoretical Physics, proceedings of the XIII International Conference, Shumen, Bulgaria, 1984, edited by H.D. Doebner and T.D. Palev (World Scientific, Singapore, 1986); J. Jurkiewicz and A. Krzywicki, Phys. Lett. 148B, 148 (1984).

${ }^{18}$ J.H. Schwarz, Phys. Rep. 89, 223 (1982); M.B. Green, Surv. High Energy Phys. 3, 127 (1983).

${ }^{19}$ O. Alvarez, Berkeley Report No. UCB-PTH-85/50 (unpublished). See also L. Alvarez-Gaume and E. Witten, Nucl. Phys. B234, 269 (1984); E. Witten, in Anomalies, Geometry, and Topology, proceedings of the Symposium, Argonne, Illinois, 1985, edited by W.A. Bardeen and A. White (World Scientific, Singapore, 1985).

${ }^{20}$ I.B. Frenkel, J. Lepowsky, and A. Meurman, in Proceedings of the 1982 Montreal Conference on Finite Group Theory, edited by J. McKay (Lecture Notes in Mathematics) (Springer, New York, 1984); Proceedings of a conference on Vertex Operators in Mathematics and Physics, publications of the Mathematical Sciences Research Institute No. 3 (Springer, New York, 1984).

${ }^{21}$ P. Goddard, D.I. Olive, and A. Schwimmer, Report No. Imperial/TP/84-85/23, 1985 (unpublished). 